\title{
Structure of stable binary neutron star merger remnants: Role of initial spin
}

\author{
W. Kastaun \\ Max Planck Institute for Gravitational Physics (Albert Einstein Institute), \\ Callinstr. 38, 30167 Hannover, Germany \\ Leibniz Universität Hannover, Institute for Gravitational Physics, \\ Callinstr. 38, 30167 Hannover, Germany \\ Physics Department, University of Trento, via Sommarive 14, I-38123 Trento, Italy \\ INFN-TIFPA, Trento Institute for Fundamental Physics and Applications, \\ via Sommarive 14, I-38123 Trento, Italy \\ R. Ciolfi \\ INAF, Osservatorio Astronomico di Padova, Vicolo dell'Osservatorio 5, I-35122 Padova, Italy \\ INFN-TIFPA, Trento Institute for Fundamental Physics and Applications, \\ via Sommarive 14, I-38123 Trento, Italy \\ Physics Department, University of Trento, via Sommarive 14, I-38123 Trento, Italy
}

\author{
A. Endrizzi \\ Physics Department, University of Trento, via Sommarive 14, I-38123 Trento, Italy \\ INFN-TIFPA, Trento Institute for Fundamental Physics and Applications, \\ via Sommarive 14, I-38123 Trento, Italy \\ B. Giacomazzo \\ Physics Department, University of Trento, via Sommarive 14, I-38123 Trento, Italy \\ INFN-TIFPA, Trento Institute for Fundamental Physics and Applications, \\ via Sommarive 14, I-38123 Trento, Italy
}

(Received 13 December 2016; revised manuscript received 28 May 2017; published 30 August 2017)

We present general relativistic numerical simulations of binary neutron star (BNS) mergers with different initial spin configurations. We focus on models with stars of mass $1.4 M_{\odot}$ each, which employ the equation of state (EOS) by Shen, Horowitz, and Teige, and which result in stable NSs as merger remnants. For comparison, we consider two irrotational equal mass $\left(M=1.35 M_{\odot}\right)$ and unequal mass $\left(M=1.29,1.42 M_{\odot}\right)$ BNS models using the APR4 EOS, which result in a supramassive merger remnant. We present visualizations of the fluid flow and temperature distribution and find a strong impact of the spin on vortex structure and nonaxisymmetric deformation. We compute the radial mass distribution and the rotation profile in the equatorial plane using recently developed measures independent of spatial gauge, revealing slowly rotating cores that can be well approximated by the cores of spherical stars. We also study the influence of the spin on the inspiral phase and the gravitational wave $(\mathrm{GW})$ signal. Using a newly developed analysis method, we further show that gravitational waveforms from BNS mergers can exhibit one or more phase jumps after merger, which occur together with minima of the strain amplitude. We provide a natural explanation in terms of the remnant's quadrupole moment, and show that cancellation effects due to phase jumps can have a strong impact on the GW power spectrum. Finally, we discuss the impact of the spin on the amount of ejected matter.

DOI: 10.1103/PhysRevD.96.043019

\section{INTRODUCTION}

After the recent detections of gravitational waves (GWs) from binary black hole $(\mathrm{BBH})$ merger events by the LIGO detectors [1,2] finally opened the era of GW astronomy, there is hope to detect also GWs from binary neutron star (BNS) mergers in the near future. The main uncertainty is the frequency of such events inside the detection horizon; see $[3,4]$ for observational upper limits and a review of current estimates.
Modeling the waveforms of BNS mergers is more complex than for the $\mathrm{BBH}$ case. During the late inspiral, there can be tidal effects that depend on the NS deformability (see e.g. [5-7]) which in turn depends on the equation of state (EOS) for cold matter at supranuclear densities. This might help to infer information about the latter. However, NSs can also have spins. Although the spin cannot be as large as for BHs, recent studies [8-11] have found a significant impact on the late inspiral, 
comparable to tidal effects. It is however unclear how rapidly NSs in binaries close to merger typically rotate. The component J0737-3039A of the famous double pulsar system [12] reaches $44 \mathrm{~Hz}$. Using the current spindown and assuming a constant braking index, it was estimated in $[13,14]$ that the spin at merger will still be $\approx 37 \mathrm{~Hz}$. However, too few BNS systems have been observed to exclude larger spins. The possibility of initial NS spin therefore needs to be considered for the interpretation of BNS GW signals.

We note that there are only a few studies on the spin since suitable methods of computing initial data have been developed only recently. In $[10,15,16]$ solutions of isolated rotating stars were superposed. In [10], the constraint violating data was evolved, while in $[15,16]$ the constraint equations were solved again after the superposition. In [17], the Bowen-York formalism for BBHs was extended to BNS. In [8,9], a rotational velocity fields was added manually to irrotational binary models and the constraint violating initial data was evolved with a constraint-damping formalism. In $[11,14,18-20]$ fully consistent initial data was used, computed with the method developed in [13,21]. Most studies considered aligned spins, with the exception of $[14,18]$ who present precessing systems as well. Further, [9] is the only study of spinning stars that also uses threeparametric tabulated nuclear physics EOSs including thermal and composition effects.

In contrast to the BBH case, the waveforms from BNS mergers do not necessarily terminate at merger. If the total mass is below a certain threshold [22,23], the merger results in a strongly deformed remnant that emits GWs with strain amplitude comparable to the last orbit, but higher frequencies. This postmerger signal carries a wealth of information. The most basic quantity provided by future detections of a postmerger signal will be a lower limit on the lifetime of the remnant (lower limit because the signal amplitude can simply fall below detection threshold without collapse to a BH). The lifetime of the remnant depends on its mass. Roughly, one classifies merger remnants as hypermassive if the total mass of the initial binary is above the maximum mass supported by uniform rotation (but below the threshold for prompt collapse during merger), and supramassive if the mass is above the maximum mass for a nonrotating NS (Tolman, Oppenheimer, Volkoff (TOV) solution). If it is even lighter, the remnant is a stable NS. All the above thresholds depend strongly on the EOS.

An observational constraint of the remnant lifetime together with the total mass should also constrain the EOS. The above classification is however only a rough indication, since the actual remnant lifetime depends on many factors. The first factor is the mass of the disk that forms around the remnant, and its accretion time scale. Another important factor is the rotation profile of the remnant. A common notion of hypermassive stars is that differential rotation with a rapidly rotating core (as described in [24]) is preventing immediate collapse, and that collapse occurs when the differential rotation inside the remnant falls below a critical level. Similarly, supramassive remnants are thought to be stabilized by uniform rotation of the core, and to collapse once sufficient angular momentum has been carried away by GW and/or magnetic braking. A mounting number of studies [9,25-27] find however that the cores of hypermassive and supramassive merger remnants rotate more slowly than the outer layers. In [9], it was shown that the outer layers can approach Kepler velocity, hence reducing the pressure onto the core and avoiding collapse. In this scenario, the lifetime depends on the angular momentum balance of those outer layers. Only one study [9] has addressed the impact of initial NS spin on the rotation profile so far, for the case of two hypermassive models. In this work, we study the same for a model in the stable remnant mass range.

The second feature likely to be observed by GW astronomy is the dominant frequency of the early postmerger stage $(<20 \mathrm{~ms})$, where the signal is still strong. This frequency is determined by the remnant properties, which are mainly given by mass and EOS, but might also be influenced by mass ratio and spins. So far, there is no robust model for the early remnant, which necessitates nonlinear numerical simulations. In a recent publication [26], we studied the fluid flow of a remnant in the stable mass range in detail and found that directly after merger the fluid flow cannot be described as simple differential rotation. In a suitable rotating frame, we found a strong, but slowly varying density deformation, with a fluid flow that contained two large vortices which rotated against each other before merging into one vortex. Even at this point the flow was not simple differential rotation; we observed large secondary vortices, phase locked with the main density deformation and also related to hot spots. We will repeat the analysis of the irrotational model shown in [26] for cases with different aligned NS spin configurations.

The interaction between vortices, hot spots, and the nonaxisymmetric deformation of the remnant is not well understood yet. One open question is to what degree the changes of the remnant deformation are caused by the dynamics of secondary vortices, as opposed to angular momentum loss. In any case, those findings suggest that linear perturbation theory is not a suitable tool to describe such systems, in particular the main GW frequency. A robust model of the remnant is required in order to constrain the EOS from observations of postmerger GW frequency. There are phenomenological studies [28-32] linking the remnant oscillation frequencies to properties of the initial stars or to the tidal coupling constant of the binary [33], but those studies do not consider the initial NS spin. 
The analysis of BNS waveforms is complicated by rapidly changing frequencies of the main signal. The phase velocity often shows pronounced peaks during and/or shortly after merger, which often correlate with sudden minima in the amplitude. In a previous work [9], we interpreted the frequency maximum during merger as a consequence of the maximum compression. In this work, we will discuss another effect that naturally leads to sudden frequency minima and maxima without requiring sudden physical changes, namely near-zero crossings of the remnant quadrupole moment in a rotating frame. For this, we developed a new method to decompose the complex-valued GW strain obtained in numerical simulations. In some cases, the strain amplitude also shows a more complex evolution than simple damping. In particular, one can often observe temporary minima. One might attribute those to unstable growing modes (this was suggested e.g. in [34] as a possible explanation for temporary minima encountered in their simulation results). Without identification of the mode, unstable mode growth remains speculative. It is also not the only explanation: we will discuss rearrangements of the fluid flow as another possible explanation for similar minima encountered in our simulations.

This paper is organized as follows. In Sec. II A, we introduce our models and discuss potential shortcomings as well as a method to quantify the spin of the stars in general relativity (GR). In Sec. II B, we describe the evolution code and diagnostic quantities used. Our new GW analysis methods are described in Secs. II C and II D. The numerical results are presented in Sec. III. Unless noted otherwise, we use units $G=c=M_{\odot}=1$.

\section{SETUP}

\section{A. Models}

In this work, we mainly investigate an equal mass binary with the Shen-Horowitz-Teige (SHT) EOS [35,36] and four different configurations for the initial spin of the NS: irrotational (IRR), both aligned (UU), both antialigned (DD), and aligned-antialigned (UD) with respect to the orbital angular momentum. The irrotational model has already been investigated in [26]. The parameters of the models are listed in Table I. Due to the large maximum baryonic mass $\left(3.33 M_{\odot}\right)$ of TOV stars supported by the SHT EOS, the merger remnant will be a stable NS. This is a corner case, but might well occur in nature; at least it is not ruled out by any observation yet.

In order to assess the generality of some results not related to spin, we also consider two irrotational models with a piecewise polytropic approximation [37] of the APR4 EOS [38]. During evolution, a $\Gamma$-law thermal part with $\Gamma_{\text {th }}=1.8$ is added. Both models are in the supramassive mass range for the APR4 EOS. The unequal mass model is the one studied in [25], and the equal-mass model is presented in detail in a different publication [39]; here
TABLE I. Initial data parameters. $M_{b}^{\text {tot }}$ is the total baryonic mass of the systems, $M_{g}$ is the gravitational mass of each star at infinite separation, and $d$ the initial proper separation. $F_{R}$ is the rotation rate of the stars with respect to the irrotational case, and $\xi$ the corresponding dimensionless angular momentum. A positive sign means aligned with the orbital angular momentum, negative means antialigned.

\begin{tabular}{lccccrr}
\hline \hline Model & EOS & $M_{b}^{\text {tot }}\left[M_{\odot}\right]$ & $M_{g}\left[M_{\odot}\right]$ & $d[\mathrm{~km}]$ & $F_{R}[\mathrm{~Hz}]$ & \multicolumn{1}{c}{$\xi$} \\
\hline SHT_IRR & SHT & 3.03 & 1.40 & 57.6 & 0 & 0 \\
SHT_UU & SHT & 3.03 & 1.40 & 57.6 & +164 & +0.125 \\
SHT_UD & SHT & 3.03 & 1.40 & 57.6 & \pm 164 & \pm 0.125 \\
SHT_DD & SHT & 3.03 & 1.40 & 57.6 & -164 & -0.125 \\
APR4_EM & APR4 & 2.98 & 1.35 & 59.0 & 0 & 0 \\
APR4_UM & APR4 & 3.01 & $1.29,1.42$ & 59.0 & 0,0 & 0,0 \\
\hline \hline
\end{tabular}

we analyze the same simulation data in comparison to our new results.

All models are produced using the LORENE code $[40,41]$. In order to add initial spin, we follow the receipt in $[8,9]$. In short, we add a rotational velocity field to the irrotational initial data. The velocity field is obtained by scaling the residual velocity field in the coorbiting frame by a constant factor.

This method of adding spin has mainly three potential sources of error. First, we violate the constraint equations of GR. In particular, the metric is completely unchanged on the initial time slice, thus missing spin related effects such as frame dragging. Second, the rotational velocity field added is not per se guaranteed to conserve the linear momentum of the stars. Such an error would manifest itself as differences of the orbital eccentricity between the different spin configurations. Finally, since we do not change the mass density, hydrostatic equilibrium is not respected, which will lead to oscillations of the NSs during inspiral. As we will show in Sec. III A, the constraint violations diminish quickly during the evolution, the oscillations are small from the start, and the eccentricities are comparable.

In GR, it is difficult to quantify the NS spin, in particular for constraint-violating initial data. In a previous work [9], we used a volume integral formulation [40] of Arnowitt, Deser, Misner (ADM) angular momentum that is based on matter terms and has a similar form as the Newtonian formula. We then computed the difference between a spunup model and the irrotational one. For this work, we use the isolated horizon (IH) formalism $[42,43]$ instead. We recall that this formalism is designed for measuring the spin of BHs using only spacetime quantities on the apparent horizon (see [44] for a review). A recent publication [18] has demonstrated that the IH spin measure is also useful for NSs in binaries. Instead of apparent horizons, the formulas of the IH framework are applied to spherical surfaces around each of the stars. In the following, we will refer to the resulting measures as quasilocal (QL) spin. 
Note that the method still does not involve the stress-energy tensor, but only the 4-metric. Since our method of adding spin does not change the metric at all, the QL spin remains unchanged initially. The spins given in Table I therefore refer to the time $0.5 \mathrm{~ms}$ after the start of the simulation. As will be shown in Sec. III A, the spacetime has adapted to the rotating fluid at this time, i.e. the inconsistency described above (which is caused by constraint violations) has fallen to a tolerable level. We report the QL angular momentum $J$ both as a rotation rate $F_{R}=J /(2 \pi I)$ and as dimensionless value $\xi=J / M_{g}^{2}$, where $I$ and $M_{g}$ are moment of inertia and gravitational mass of the stars in isolation (for the SHT models, $I=48.45 M_{\odot}^{3}$ ). For comparison, we also computed the difference of the ADM angular momentum for the SHT models, as in [9], and found values $\xi= \pm 0.119$, i.e. the two different measures agree within $5 \%$.

\section{B. Numerical evolution}

For the numerical evolution of the spinning SHT models, we use the same numerical codes as for the evolution of the irrotational SHT model, described in [26]. The hydrodynamic evolution is performed by the WHISKYTHERMAL code which employs finite-volume high resolution shock capturing methods in conjunction with the HLLE approximate Riemann solver and the piecewise parabolic reconstruction method. We use a three-parameter tabulated EOS including thermal effects and composition in terms of the electron fraction (see [45] for technical details). We do not consider magnetic fields and neutrino radiation, and the electron fraction is passively advected along the fluid. Vacuum regions are treated numerically by enforcing a minimum density (artificial atmosphere) of $6 \times 10^{7} \mathrm{~g} / \mathrm{cm}^{3}$. Note this is a relatively high density necessitated by the extent of the SHT EOS table in use. Regions covered by artificial atmosphere are not included in the stress energy tensor used as source term for the spacetime evolution. During the inspiral, we also enforce adiabatic evolution, activating the full thermal evolution shortly before the stars touch (see [26]).

The spacetime is evolved with the McLachlan code [46], which is part of the Einstein Toolkit [47]. For all SHT models, spinning and irrotational, we chose the conformal and spatially covariant Z4 evolution scheme (CCZ4) described in $[48,49]$, which has constraint damping capabilities that are advantageous when evolving constraintviolating initial data. As gauge conditions, we use the $1+\log$-slicing condition [50] for the lapse function and the hyperbolic $\Gamma$-driver condition [51] for the shift vector. At the outer boundary, we use the Sommerfeld radiation boundary condition. To extract the multipole components of the Weyl scalar $\Psi_{4}$, we use the modules Multipole and WEYLSCAL4 described in [47].

All codes are based on the Cactus Computational Toolkit infrastructure. The time evolution of matter and spacetime is coupled using the Method of Lines (MoL) with fourth order Runge-Kutta time integration. We also use BergerOliger moving-box mesh refinement implemented by the CARPET code [52]. In detail, we use six refinement levels. During inspiral, the two finest ones consist of cubical boxes following the movement of the two stars (each completely contained in the corresponding smallest box). Shortly before merger, they are replaced by fixed levels centered around the origin, with an edge length of $60 \mathrm{~km}$ for the finest box. The finest grid spacing during the whole simulation is $295 \mathrm{~m}$ and the outer boundary is located at $945 \mathrm{~km}$. Finally, we use reflection symmetry across the orbital plane. For tests of the code, we refer to $[45,49]$.

The two models with the APR4 EOS were evolved with a slightly different setup, as described in $[25,39]$. The hydrodynamics was evolved using the WHISKYMHD code (with zero magnetic field) using a piecewise polytropic EOS, and without enforcing adiabatic evolution during inspiral. The artificial atmosphere in this case had a lower density of $6 \times 10^{6} \mathrm{~g} / \mathrm{cm}^{3}$. The spacetime was evolved with the MCLaChlan code, but using the Baumgarte, Shibata, Shapiro, Nakamura (BSSN) formulation [53-55]. The outer boundary was located at 794 and $1250 \mathrm{~km}$ for models APR4_UM and APR4_EM, respectively, and the finest resolution was $221 \mathrm{~m}$ for both. In terms of stellar radius, this resolution is the same as for the less compact SHT models (the grid spacing is $\approx 1 / 40$ of stellar equatorial coordinate radius or $1 / 50$ of circumferential radius). We will therefore use resolution studies carried out in $[25,39]$ for magnetized versions of the models above to estimate the numerical error for the SHT simulations. We caution that in $[25,39]$ we could not demonstrate convergence for most quantities, likely because the lowest resolution was too low, and estimated errors are based on the standard and high resolution runs only.

We also employ the diagnostic measure for the remnant properties introduced in [26]. At regular time intervals during the evolution, we create 1D histograms of both proper volume and baryonic mass contained in the grid cells, using bins of logarithmic rest-frame mass density. From the histograms, we then compute the total proper volume $V$ and the total baryonic mass $M_{b}$ contained inside isodensity surfaces. This allows us to compute massvolume relations that are still meaningful for the strongly deformed merger remnants and which are defined independent of the spatial gauge conditions. Note there is still a dependence on the time slicing, so when comparing different models the extrinsic curvature should be compared as well. We expect the $1+\log$ gauge used here to drive the system towards maximal slicing (the residual extrinsic curvature was not measured, however). We also define a "volumetric radius" $R_{V}$ for the isodensity surfaces as the radius of a Euclidean sphere with equal volume, and from this a compactness measure $C=M_{b} / R_{V}$. To define remnant properties without referring to some arbitrary density or radius cutoffs, we use the notion of the bulk, defined as 
the interior of the isodensity surface with maximum compactness $C$. Bulk mass and volume are then defined as baryonic mass and proper volume of the bulk. For further details, see [26].

For comparison, we computed the new bulk measures for sequences of stable TOV stars with the APR4 [38], H4 [56], MS1 [57], and SHT EOS [35,36], and $M_{g}$ between $1 M_{\odot}$ and the maximum. For those, the bulk (on $K=0$ time slices) contains $96.5 \%-98.5 \%$ of the total baryonic mass, thus motivating the name. The new compactness is less than the standard compactness (gravitational mass divided by circumferential surface radius), by a factor $0.85-0.93$. Bulk mass and compactness are strictly monotonic functions of total baryonic mass and standard compactness, respectively.

\section{Improved GW extraction}

To extract the GW signal, we use the standard approach of decomposing the Weyl scalar $\Psi_{4}$ into spin weighted spherical harmonics on a sphere with large radius close to the outer boundary of the computational domain (916, 1181 , and $738 \mathrm{~km}$ for the SHT, equal- and unequal-mass APR4 models, respectively). The tetrad choice for the computation of $\Psi_{4}$ is the one given in [47]. We do not extrapolate to infinity since we are only interested in a qualitative analysis. To compute the strain, $\Psi_{4}$ has to be integrated in time twice, which will amplify any offset present in the numerical waveform drastically. There are two standard methods in use to remove those offsets (see also the discussion in [58]). The most straightforward one is to fit a linear or quadratic function to the strain to determine the nonoscillatory part and then subtract it. However, we found this approach insufficient for our purposes. We are particularly interested in the behavior of the phase near regions where the strain amplitude temporarily becomes small. It is thus important that the offset is even smaller, which cannot be achieved by a simple fit since it will be dominated by the large amplitude parts.

The second common approach is the fixed frequency integration (FFI) method [59], where the integration is performed in frequency space by means of a Fourier transformation and the suppression of the low frequency part of the spectrum. This method also turned out insufficient. The problem is that the Fourier spectrum of a linear drift has high frequency components because of the finite length of the waveform, overlapping the actual signal. In practice, this causes strong drifts near the beginning and end of the resulting waveform.

To overcome these issues, we developed a new integration method applicable even to short waveforms. The main idea is to first compute a local approximation to the integral based on the assumption of slowly changing amplitude and frequency. The estimate is then subtracted from the numerically integrated function. We then fit a slowly varying function to the difference, instead to the signal itself. Due to varying frequency and amplitude, the oscillatory part does not cancel out completely when fitting to the latter. The key advantage of fitting the former is that the residual oscillatory part is much smaller, thus reducing the impact on the drift correction.

In detail, the method works as follows. Given complex functions $z(t)=\dot{g}(t)$, we first compute the continuous phase $\phi_{z}$ such that $z(t)=z_{a}(t) e^{i \phi_{z}(t)}$, with $z_{a} \in \mathbb{R}$. Next, we compute a smoothed phase $\phi$ by convolution of $\phi_{z}$ with a Gaussian kernel of width $\sigma_{s}$, where $\sigma_{s} \bar{\omega}=4 \pi$, and $\bar{\omega}$ is the average phase velocity of $\phi_{z}$. We require that $\phi$ is strictly increasing as a function of time (or strictly decreasing, we assume the former without loss of generality). Next, we express $z$ and $g$ as $z(\phi)=z_{c}(\phi) e^{i \phi}$ and $g(\phi)=g_{c}(\phi) e^{i \phi}$, and define $\omega \equiv \dot{\phi}$. The phases of the complex-valued amplitudes $z_{c}$ and $g_{c}$ change much more slowly than $\phi$ itself since we removed the main oscillation. By combining the equations $z(t)=\dot{g}(t)$ and $\dot{z}(t)=\ddot{g}(t)$, neglecting the second time derivative of $g_{c}$, and then rewriting time derivatives in terms of $\phi$-derivatives and $\omega$, we obtain an estimate $\hat{g}$ for $g$, given by

$$
\hat{g}=\hat{g}_{c} e^{i \phi}, \quad \hat{g}_{c}=\frac{\frac{d z_{c}}{d \phi}-i z_{c}}{\omega+i \frac{d \omega}{d \phi}} .
$$

We then compute the integral

$$
g\left(t_{1}\right)=\int_{t_{0}}^{t_{1}} z(t) \mathrm{d} t=\int_{\phi_{0}}^{\phi_{1}} \frac{z(\phi)}{\omega(\phi)} \mathrm{d} \phi
$$

numerically (using trapezoidal integration). Next, we define the residual $\delta g(\phi)=g(\phi)-\hat{g}(\phi)$. In order to obtain the nonoscillatory part of $\delta g$, we fit a cubic spline $s$ with $N / m$ nodes regularly spaced in $\phi, N$ being the number of complete wave cycles in the signal. Note that any oscillating contribution to $s$ will have frequencies lower by $1 / \mathrm{m}$ with respect to the actual signal. In this work, we chose $m=4$. The final, drift-corrected result is simply $g-s$.

We apply this integration method twice to obtain the GW strain $h$ from $\Psi_{4}$. Comparing the method to the aforementioned standard methods, we observed a strong reduction of artifacts near the boundaries, in particular for very short waveforms. We also found a much better alignment of low-amplitude parts of the signal. For our longest waveform, the phase velocity in the decaying tail remains usable longer than for the old methods, although the phase velocity still becomes noisy when the amplitude becomes very small. This seems to be partly because the signal is not dominated by a single mode anymore, rendering the phase velocity meaningless. In any case, the GW amplitude at this point is not relevant for GW astronomy anymore.

After a perfunctory comparison to strains obtained using an implementation [47] of the Moncrief formalism [60] showed no obvious problems, we designed an analytic 
expression as a test signal for the new method in order to measure its accuracy. In detail,

$$
\begin{gathered}
h(t)=A(t) e^{i \phi(t)}+B t^{2} \\
A(t)=\cos \left(\frac{3}{2} \pi \tanh \left(\frac{t}{\tau_{1}}\right)\right) \\
\frac{1}{2 \pi} \dot{\phi}(t)=F(t)=F_{1}+\frac{F_{2}}{1+e^{-t / \tau_{2}}} .
\end{gathered}
$$

The amplitude goes to zero for $|t| \gg \tau_{1}$, and has three extrema, separated by two zero crossings. The instantaneous frequency increases from $F_{1}$ for $t \ll-\tau_{2}$ to $F_{1}+F_{2}$ for $t \gg \tau_{2}$. Moreover, the second derivative of $h$ has an offset $2 B$ in addition to the oscillatory part. We picked parameters $B=300 \mathrm{~s}^{-2}, F_{1}=0.5 \mathrm{kHz}, F_{2}=2 \mathrm{kHz}, \tau_{1}=6 \mathrm{~ms}$, $\tau_{2}=4 \mathrm{~ms}$. Next, we analytically computed the second derivative of $h$, sampled it on the interval $-15 \ldots 15 \mathrm{~ms}$, and applied the new integration method two times. Since the method is supposed to remove the nonoscillatory drift, we compare the result to the exact strain for $B=0$. For this test, we find that the $L_{1}$-norm of the error of $h$ is around $7 \times 10^{-4}$ of the maximum amplitude, and 3\% of the $L_{1^{-}}$ norm of the offset term $B t^{2}$. The $L_{1}$-norm of the phase error is around $0.002 \mathrm{rad}$.

For comparison, we also tested the FFI with a cutoff frequency of $F_{1}$. We find that the error of $h$ is 6.5 times larger than for the new method, and that the phase error is 18 times larger, both mainly due to a large drift near the boundaries of the given time interval. Note however that the boundary effects affecting the FFI method can be reduced by applying a window function before the Fourier transform, at the cost of significantly reduced usable signal length. We further note that also the polynomial fit method can be improved by applying a high-pass Butterworth filter, as shown in [34,58]. In general, it will depend on the use case which method is most suitable.

\section{Analyzing phase jumps in the GW signal}

While for BBH mergers the phase of the GW signal is relatively smooth, binary NS waveforms often exhibit strong peaks in the phase velocity. Examples will be shown in Sec. III D. In some cases, they amount to a phase shift by $\pi$. Those phase jump events also seem to correlate with minima in the amplitude. This points to a phenomenon called overmodulation in the context of signal analysis. Overmodulation occurs for amplitude-modulated signals of the type $z(t)=a(t) e^{i \omega t}$, when the modulation amplitude $a \in \mathbb{R}$ crosses zero. This corresponds to an instantaneous phase shift by $\pi$ and also leads to a nondifferentiable signal amplitude $|a|$. An introduction to overmodulated signals and a method for their decomposition is given in [61], which is however limited to real-valued signals. We slightly generalize this idealized case by allowing $a$ to be complex valued, with a small imaginary part, and instead of the zero crossing we let $a$ pass close to the origin of the complex plane. The phase will then change rapidly and the amplitude $|a|$ will have a minimum where the second derivative is large. The larger the imaginary part, the larger the minimum amplitude and the slower the phase transition will occur.

Note that in the frequency domain, energy is removed from the carrier frequency and occurs as sidebands for overmodulated signals. This is easy to understand, since parts of the signal cancel each other out because their phases are inverted with respect to each other. It is therefore important to consider overmodulation in the context of GW astronomy, where a large part of the analysis is carried out in frequency space. We will discuss the physical causes for overmodulation of GWs in Sec. III D. In the following, we present an algorithm for the detection and removal of phase jumps.

In order to detect possible phase jumps in a signal $z(t)$, we first compute a smoothed angular velocity $\omega$ from the phase velocity $\omega_{z}$ of $z$, using convolution with a Gaussian kernel of width $\sigma=4 \bar{P}$, where $\bar{P}=2 \pi / \bar{\omega}$, and $\bar{\omega}$ is the time average of $\omega_{z}$. We use the local maxima of $\eta=$ $\left|\omega_{z}-\omega\right|$ as candidates for phase jumps. We ignore maxima below a cutoff $\eta<0.1 \bar{\omega}$, and local maxima located closer than $2 \bar{P}$ to a larger local maximum. We then try to model each remaining candidate as a phase jump event described by the generic function $\delta \phi_{j}(t)=\arctan \left(k\left(t-t_{j}\right)\right)$, which corresponds to an amplitude passing close to zero in the complex plane along a line with constant speed. The derivative is given by

$$
\omega_{j}(t)=\frac{d}{d t} \delta \phi_{j}(t)=\frac{k_{j}}{1+k_{j}^{2}\left(t-t_{j}\right)^{2}} .
$$

We then fit $\omega_{z}(t)$ with the function

$$
\omega_{f}(t)=\omega_{j}(t)+k_{1 j}+\left(t-t_{j}\right) k_{2 j},
$$

using fit parameters $k_{j}, k_{1 j}, k_{2 j}, t_{j}$. Note $\omega_{j}$ has a very distinct shape near $t_{j}$, which sets it apart from other frequency fluctuations, e.g. caused by strong radial oscillations. It also decays rapidly away from $t_{j}$. We therefore limit the size of the fit interval $\left[t_{j}-\Delta t, t_{j}+\Delta t\right]$ to a certain fraction of the width of the peak given by $\omega_{j}$. In terms of the phase shift $\alpha=\delta \phi_{j}\left(t_{j}+\Delta t\right)-\delta \phi_{j}\left(t_{j}-\Delta t\right)$ within the fit window, we chose $\Delta t$ such that $\alpha \approx \pi / 4$. Since $\delta \phi_{j}$ is unknown before the fit, we use an estimate computed from the area under the measured peak,

$$
\alpha \approx \int_{t_{j}-\Delta t}^{t_{j}+\Delta t}\left(\omega_{z}(t)-\omega_{b}(t)\right) d t,
$$

where $\omega_{b}$ models the background by linear interpolation of $\omega_{z}$ between the values at $t=t_{j}-2 \bar{P}$ and $t_{j}+2 \bar{P}$. To avoid 
misinterpreting slow variations as jumps, we require that $\Delta t<2 \bar{P}$. As a last check, we also fit a simple quadratic function to the peak and ignore the candidate if the $L_{2}$ norm of the residual is less than the one for the fit with $\omega_{f}$. The above heuristic method proved adequate to detect phase jumps for the GW strains presented in this work robustly without manual intervention.

After fitting the individual phase jumps, we compute the combined phase correction as $\delta \phi(t)=\sum_{j} \delta \phi_{j}(t)$. From this, we define a complex amplitude $z_{a}(t)=|z(t)| e^{-i \delta \phi(t)}$. The real part will exhibit a zero crossing at the jumps, while the imaginary part at the jump indicates how close to zero the signal gets. We further obtain a corrected phase velocity $\omega=\frac{d}{d t}\left(\phi_{z}-\delta \phi\right)$. This phase velocity is a better indication for the frequency of the actual oscillations of the source, since most of the contribution caused by the overmodulation is removed. When applied to the test signal described in the previous section, we find that the corrected phase velocity agrees well with the exact value $\Omega(t)$. The $L_{1}$-norm of the relative error is $0.2 \%$. The largest deviations can be found near the jumps and near the boundaries, with a maximum error below $3 \%$.

\section{RESULTS}

In the following, we present the numerical results for the models shown in Sec. II A. The key quantities are summarized in Table II.

\section{A. Inspiral}

We start by assessing the potential problems with our spinning initial data described in Sec. II A. The constraint violations for the different spin configurations are shown in Fig. 1. For the spin values considered here, the initial Hamiltonian constraint is only larger by a factor around 2.5 compared to the irrotational model, and the combined momentum constraint by a factor 8 . Thanks to the CCZ4 evolution formalism, Hamiltonian and momentum constraint violations for the spinning models further decrease to the level of the irrotational model within $1 \mathrm{~ms}$. In the later inspiral, the constraint violations as a function of time differ again, but only because there is a physical impact on the duration of the inspiral, as will be discussed later. The amount of constraint violation at merger time is very similar for the different spins.

Satisfying the constraints only means that the spacetime is consistent on the corresponding time slice; it does not imply that it represents the initial data we want, i.e. spinning NSs in quasicircular orbit. In order to measure how the metric near the stars adapted to the manually added NS rotation, we use the quasilocal spin described in Sec. II A. Figure 2 shows the QL angular momentum for the first $2 \mathrm{~ms}$ of the simulations, extracted on spherical surfaces with radius $13.3 \mathrm{~km}$ around the NS barycenters. Since we do not change the metric at all, the curves for the spinning models start at the same value as the irrotational model. Within less than $0.5 \mathrm{~ms}$, however, the spacetime near the stars apparently adjusts to the rotation of the fluid. We thus regard the system at this time as our true initial data, and the spin values reported in Table I are the differences of the QL measure to the irrotational model at $0.5 \mathrm{~ms}$.

Note that the QL spin for the irrotational model is not exactly zero initially, we find $\xi \approx 0.008$. Although there is no reason to expect it to be zero for actual irrotational binaries at finite distance, it is unknown how much of the residual spin is generated by the imperfections of the irrotational initial data. Using a different initial data code for a different model, but with comparable initial separation, [18] reported a much smaller value of $\xi=2 \times 10^{-4}$.

TABLE II. Outcome of the mergers. $M_{\mathrm{blk}}, R_{\mathrm{blk}}$, and $S_{\mathrm{blk}}$ are bulk mass, bulk radius, and bulk entropy, $\nu_{\text {max }}$ denotes the remnants maximum rotation rate in the equatorial plane, all computed $14 \mathrm{~ms}$ after the merger. $f_{\mathrm{pk}}$ is the GW instantaneous frequency at merger time, and $f_{\mathrm{pm}}$ is the frequency of the largest peak in the postmerger part of the GW power spectrum. $M_{\text {disk }}$ is the bound mass outside a coordinate sphere with proper volumetric radius $26 \mathrm{~km}, M_{60}$ is the bound mass outside $r>60 \mathrm{~km}$, both measured at $t=14 \mathrm{~ms}$ after merger. Finally, $M_{\mathrm{e}}$ and $v_{\infty}$ are our best estimates for the total mass of ejected matter and its average velocity at infinity. $M_{\mathrm{e}}^{\text {fix }}$ is the ejected mass measured using a surface with fixed radius $222 \mathrm{~km}$.

\begin{tabular}{|c|c|c|c|c|c|c|}
\hline Model & SHT Irr & SHT UU & SHT UD & SHT DD & APR4 UM & APR4 EM \\
\hline$M_{\mathrm{blk}}\left[M_{\odot}\right]$ & 2.44 & 2.46 & 2.57 & 2.44 & $\ldots$ & $\ldots$ \\
\hline$M_{\mathrm{blk}} / R_{\mathrm{blk}}$ & 0.220 & 0.221 & 0.223 & 0.216 & $\cdots$ & $\cdots$ \\
\hline$S_{\mathrm{blk}} / M_{\mathrm{blk}}\left[k_{\mathrm{B}} /\right.$ Baryon $]$ & 0.99 & 0.84 & 1.12 & 1.29 & $\cdots$ & $\cdots$ \\
\hline$\nu_{\max }[\mathrm{kHz}]$ & 0.95 & 0.94 & 0.96 & 0.95 & 1.64 & 1.66 \\
\hline$f_{\mathrm{pk}}[\mathrm{kHz}]$ & 1.42 & 1.45 & 1.33 & 1.41 & 2.09 & 2.12 \\
\hline$f_{\mathrm{pm}}[\mathrm{kHz}]$ & 2.02 & 2.09 & 2.18 & 1.96 & 3.30 & 3.47 \\
\hline$M_{\mathrm{disk}}\left[M_{\odot}\right]$ & 0.291 & 0.296 & 0.163 & 0.236 & $\ldots$ & $\cdots$ \\
\hline$M_{60}\left[M_{\odot}\right]$ & 0.158 & 0.159 & 0.053 & 0.106 & $\cdots$ & $\cdots$ \\
\hline$M_{\mathrm{e}}\left[M_{\odot}\right]$ & 0.0003 & 0.0011 & $<10^{-4}$ & 0.0007 & 0.0100 & 0.0126 \\
\hline$M_{\mathrm{e}}^{\mathrm{fix}}\left[M_{\odot}\right]$ & $<10^{-4}$ & 0.0009 & $<10^{-4}$ & 0.0003 & $\cdots$ & $\cdots$ \\
\hline$v_{\infty}[c]$ & 0.10 & 0.12 & 0.06 & 0.10 & $\cdots$ & $\cdots$ \\
\hline
\end{tabular}



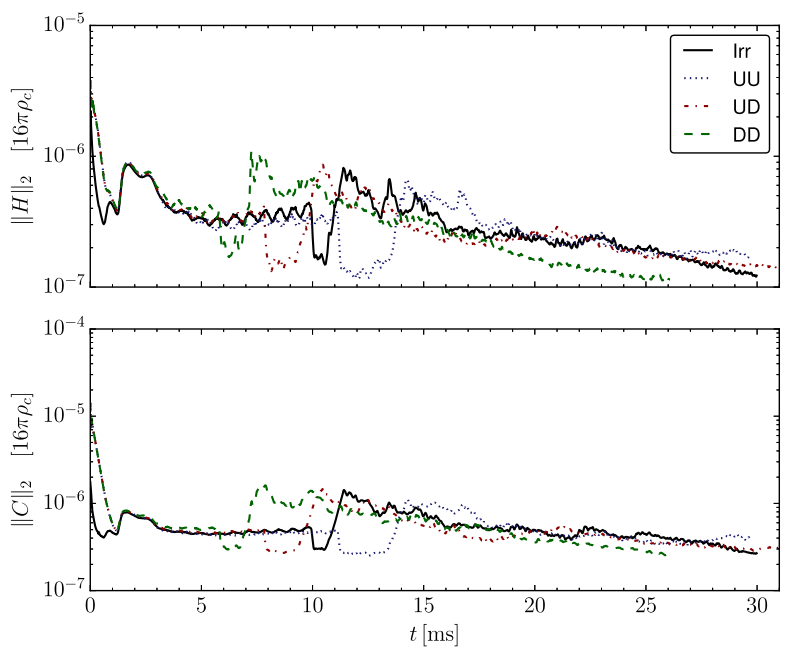

FIG. 1. Evolution of constraint violations for the SHT EOS models. Top panel: $L_{2}$-norm $\|H\|_{2}$ of the Hamiltonian constraint $H$. Bottom panel: combined $L_{2}$ norm $\|C\|_{2} \equiv\left(\sum_{i=1}^{3}\left(\left\|C_{i}\right\|_{2}\right)^{2}\right)^{1 / 2}$ of momentum constraints $C_{i}$. All constraints are normalized using the central density $\rho_{c}$ of the initial stars.

Also note that the QL spin measure exhibits a slow drift during the whole inspiral. This drift seems to be the same regardless of the NS spin, even for the irrotational model. The reason might be that it becomes more difficult to separate orbital and individual spin contributions to the metric when the stars come closer. This is however beyond the scope of this work; the issue is investigated in more detail in [11]. Here we only used the QL spin to assess the
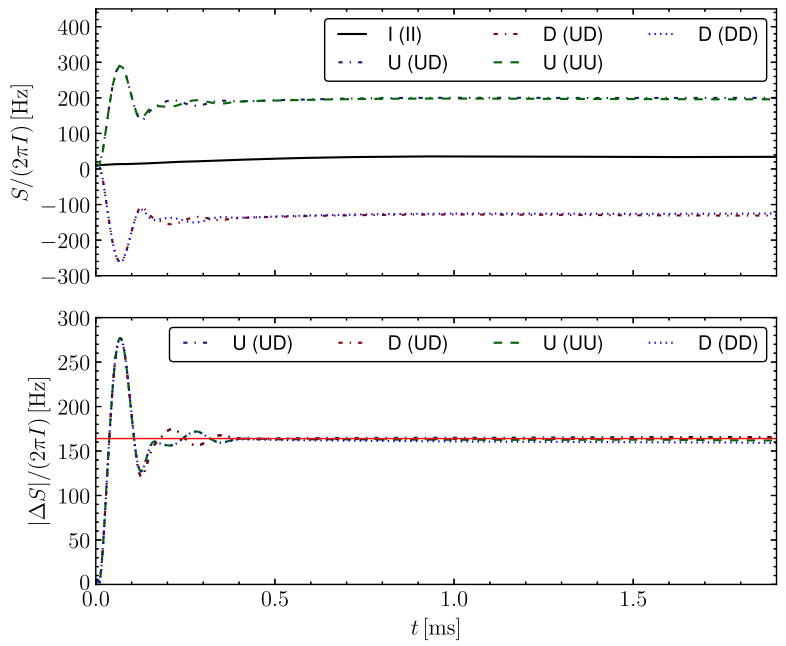

FIG. 2. Top panel: Quasilocal angular momentum $S$ of the individual stars for the SHT EOS models. $I$ is the moment of inertia of the TOV solution with the same baryonic mass. Stars with aligned, antialigned, and irrotational spin configurations are denoted U, D, and I, respectively, with the binary model they are part of given in brackets. Bottom panel: The difference of the spin to the one for the irrotational model. The horizontal line marks the nominal spin of $164 \mathrm{~Hz}$. quality of the initial data. In this regard, we also note that the NS oscillations induced by adding the spin are rather small for our cases. The central density varies by less than $0.6 \%$ for all spin configurations. For comparison, the central density oscillation amplitude for the irrotational model is around $0.2 \%$.

Figure 3 shows the proper separation between the density maxima of the NSs versus their orbital phase (measured with respect to simulation coordinates). Judging by eye, both the irrotational and the spinning models show a similar degree of eccentricity. We therefore assume the additional error in the linear momentum due to spin is unimportant, but caution that a solid error estimate would require further simulations and that, strictly speaking, all our results are valid for a slightly eccentric binary.

The number of orbits until merger however is clearly affected by the initial NS spin: increasing the spin of the NSs aligned with the orbital angular momentum prolongs the inspiral, while in the antialigned case it is accelerated. The inspiral time (time between the start of simulation and merger) follows the same trend as the number of orbits, with 7.4, 10.0, 11.3, and $14.0 \mathrm{~ms}$ for the down-down, updown, irrotational, and up-up configurations, respectively. To estimate the error due to finite resolution, we use the resolution study in [25]. For this case, second order convergence was demonstrated for the GW signal during inspiral, and we estimate a total phase error during six orbits of inspiral of around $0.6 \mathrm{rad}$. However, this just constitutes a best guess, since the resolution study was done for an irrotational unequal-mass model with a different EOS (APR4). Other differences are likely less important: the use of the BSSN formalism instead of CCZ4 should result in comparable errors, as demonstrated in [49]; although [25] evolved the magnetohydrodynamic evolution equations as well, this will, if anything, increase the error; the lower density of the artificial atmosphere is not relevant for the inspiral since the (Newtonian) dynamic pressure

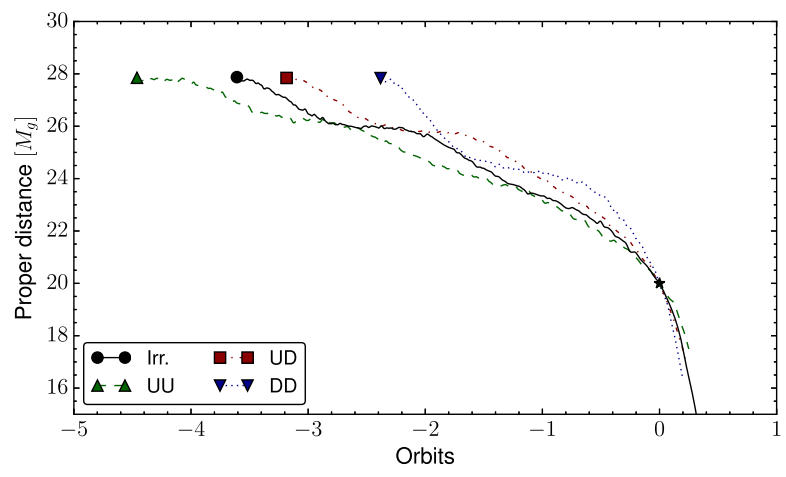

FIG. 3. Evolution of proper separation between the NSs density maxima versus orbital phase, for the SHT EOS models. The separation is given in units of the single star gravitational mass in isolation, and the curves have been aligned at a separation of $20 M_{g}$. 
obtained for the SHT simulations in this work corresponds to deceleration time scales $v / \dot{v} \gtrsim 10^{3} \mathrm{~s}$.

Our findings further corroborate this orbital hangup effect, which was already described in previous studies $[9,10,19]$ for different models and, more importantly, three different methods of adding the initial NS spin. In order to quantify the effect precisely, it would be necessary to further reduce the eccentricity, using e.g. the methods described in $[14,18,62]$. As a ballpark figure for the cases at hand, varying the initial spin between $\pm 164 \mathrm{~Hz}$ changes the length of the inspiral by two full orbits.

\section{B. Postmerger dynamics}

To get an overview of the fluid flow during and after merger, we computed the fluid trajectories in the equatorial plane in a coordinate system corotating with the $m=2$ component of the density perturbation in the equatorial plane. The latter is computed from the phase of the complexvalued $m=2$ moment of the density in the equatorial plane. Since we only want to remove the average rotation for visualization purposes, but not introduce noise in the trajectories, we smoothed the phase in time using convolution with a $2 \mathrm{~ms}$ Hanning window. The trajectories are computed by integrating backwards in time in a postprocessing step, as described in [26]. In particular, we use the coordinate system defined there to suppress deformations due to gauge effects.

In Fig. 4, we show the trajectories as a $2+1 \mathrm{D}$ spacetime diagram where two dimensions correspond to the equatorial plane. Here we compare the results for all spin configurations to the irrotational case (which was shown in a similar plot already in [26]). Removing the overall rotation is necessary in order to visualize the vortex structure of the fluid flow. We note however that there is a certain ambiguity in the choice of the rotation since during merger, the principal axes of the $m=2$ deformation are exchanged. This leads to a rotation of the plot coordinates by $90^{\circ}$ within the smoothing length, which is advantageous for the purpose of the visualization. In the following, we refer to the larger and smaller principal axes of the remnant $m=2$ deformation in the equatorial plane as $x$ and $y$ axes.

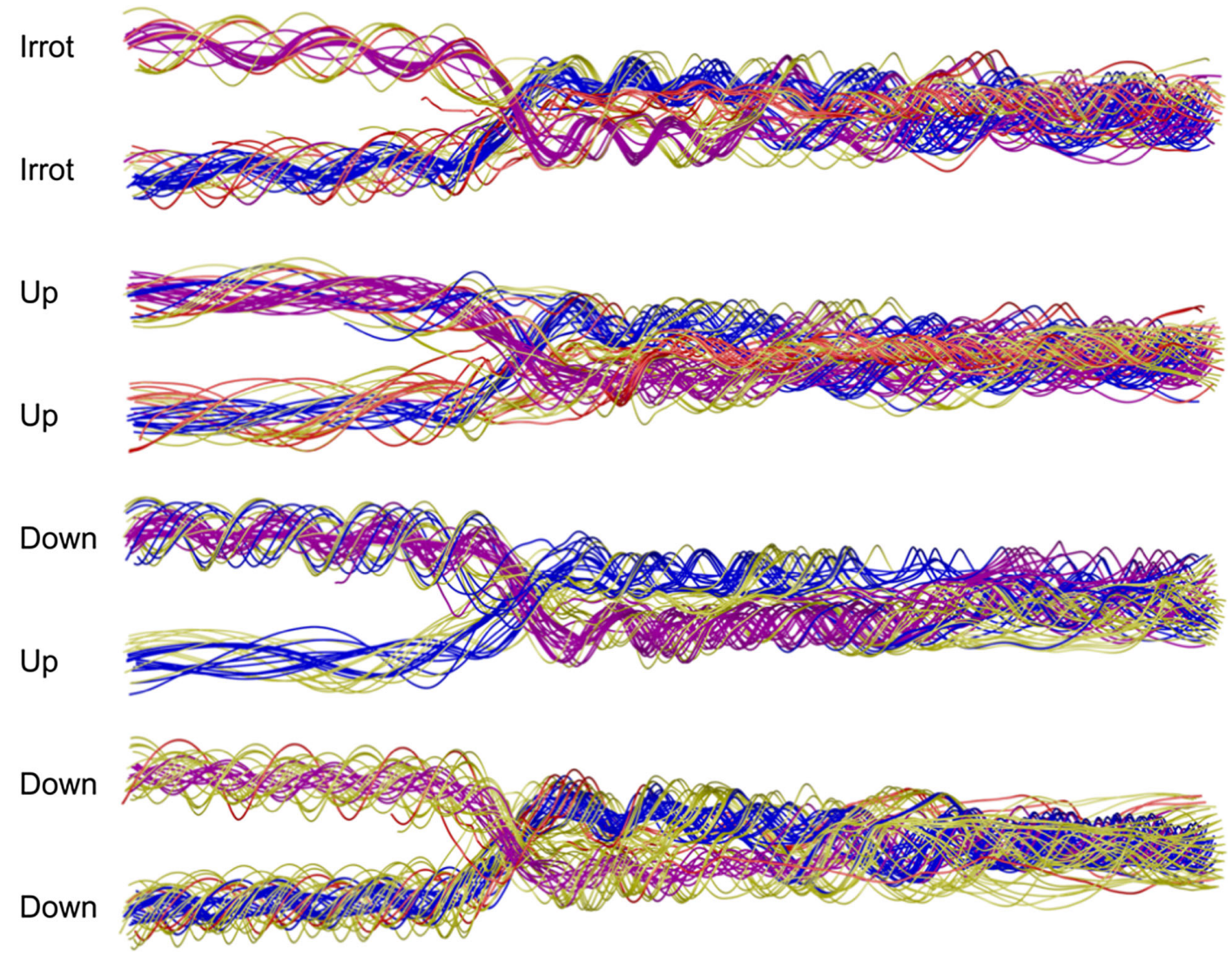

FIG. 4. Comparison of fluid trajectories in the equatorial plane for different initial spins, for the SHT EOS. The time runs from left to right and spans the interval $7 \mathrm{~ms}$ before merger to $14 \mathrm{~ms}$ after merger. The coordinates in the equatorial plane are corotating with the density deformation (see text); the larger principal axis ( $x$ axis) is pointing up. Only trajectories remaining in the remnant are shown, and a density cutoff at $10^{9} \mathrm{~g} / \mathrm{cm}^{3}$ is applied (this is the reason why some trajectories start later). Trajectories belonging to the two main vortices after merger are colored blue and violet, trajectories passing a secondary vortex in the early and late postmerger phases are colored red and yellow, respectively. 
Before we discuss the differences, we review the features common to all models. Directly after merger, the cores of the NSs have formed two large vortices which are rotating against each other. Their separation vector is oriented along the $x$ axis. This continues for several milliseconds until the two vortices slowly merge into a single larger vortex that is better described as differential rotation, but with a strong nonaxisymmetric deformation. In addition to the main vortices, we also observe smaller secondary vortices, which become more elongated over time, but are still present at the end of the simulation. Those secondary vortices are located in the half-planes $y>0$ and $y<0$, i.e. the centers are rotated around $90^{\circ}$ with respect to the density distribution. To highlight the primary vortices in the figure, we colored trajectories blue or purple if they stay in the $x<0$ or $x>0$ half-planes, respectively, during the early postmerger phase (1-5 ms after merger). Trajectories which stay in one of the half-planes $y>0$ or $y<0$ during the early (late) postmerger phase are colored red (yellow), to highlight the secondary vortices. We note that some trajectories also escape or join the vortices. All vortices are phase locked with the main density perturbation.

Note that directly after merger, we also expect a KelvinHelmholtz $(\mathrm{KH})$ instability, which is however not well resolved at the resolution used here. A high-resolution numerical study for the case of magnetized systems can be found in [63]. For our simulations, we expect that the time scale on which the two vortices merge is most likely affected, but it is difficult to estimate the numerical error.

As can be seen in Fig. 4, the general evolution outlined above is strongly influenced by the initial spin. The first difference is that the large vortices rotate with different

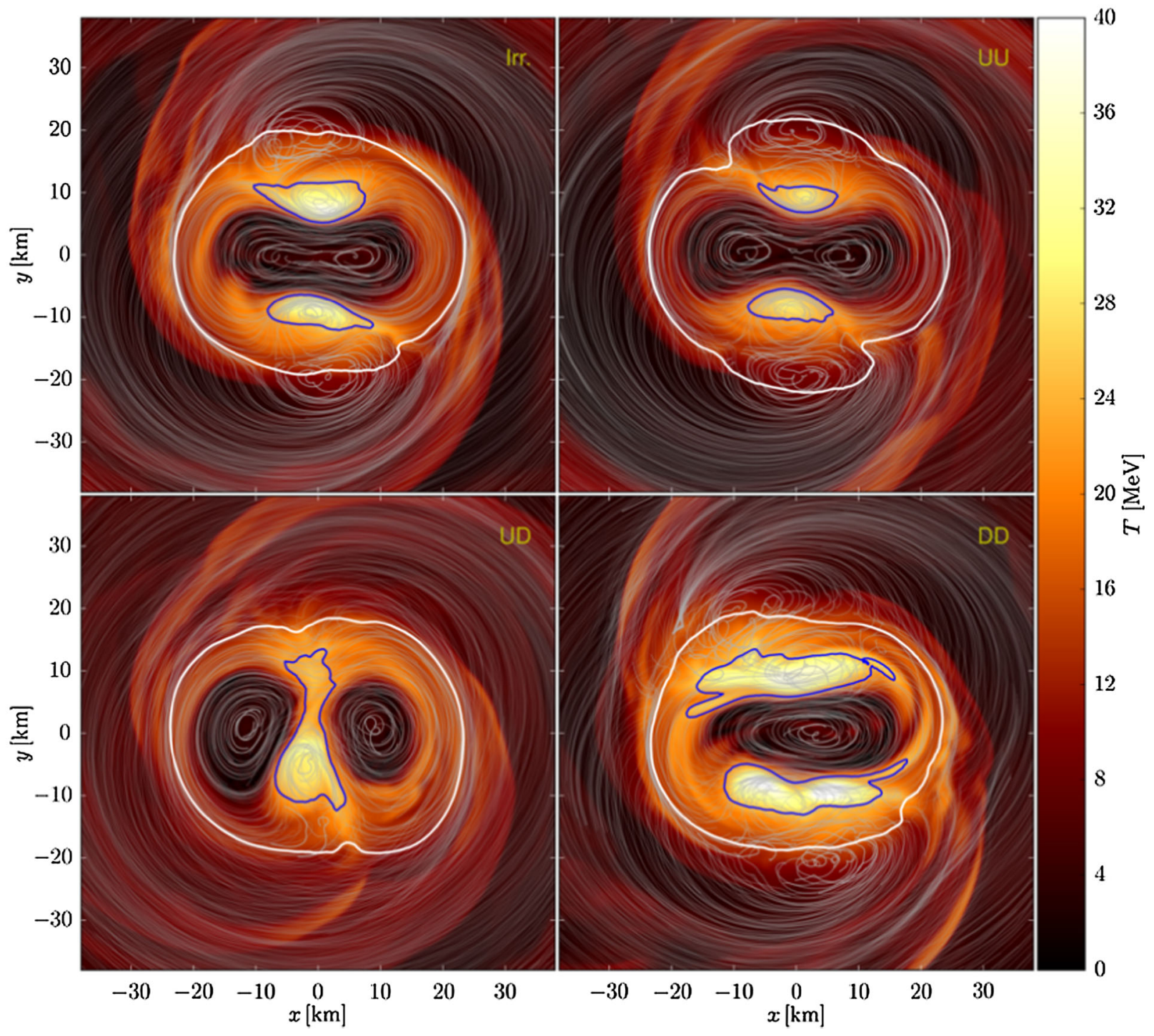

FIG. 5. Comparison of vortex structure $8 \mathrm{~ms}$ after merger for different initial spin. The temperature is shown as a color plot. The thick white line is the isodensity contour corresponding to the bulk. The blue lines are isocontours of entropy density, marking the hot spots. The thin white lines are fluid trajectories in the frame corotating with the $m=2$ density perturbation, in a time window from 7 to 9 ms after merger. 
speeds against each other, which is to be expected. Figure 4 also provides a first indication that the size and shapes of the secondary vortices are strongly affected. We will come back to this later on. It is also worth noting that the mixed spin model SHT_UD shows a strong asymmetry, apparently related to the fact that the two main vortices rotate with different speed.

Close inspection of Fig. 4 reveals an unequal number of trajectories in the two inspiraling NSs even for the $\pi$-symmetric models. This is unexpected since the seed positions at the end of the postmerger phase are also $\pi$ symmetric. Note however that the mapping from the trajectory positions at the end of the simulation to the positions at the beginning is not smooth at all. The constant churning motion and the crossing of trajectories from one vortex to another is likely to make this map almost chaotic in the sense that small changes in the initial conditions lead to large changes in the outcome. Under perfect conditions, this still cannot break the symmetry. However, numerical errors and possibly physical instabilities will introduce small asymmetries. Considering also the aforementioned selection rules for the plotted trajectories, it is hard to predict what distribution of trajectories in the inspiral phase will result even from small asymmetries. We therefore caution that Fig. 4 should be used only to visualize the overall fluid flow.

The deformation of the remnant, its thermal structure and flow patterns $8 \mathrm{~ms}$ after merger are depicted in Fig. 5 (similar movies are available in the Supplemental Material of this article [64]). At the time shown in the figure, the main vortices have already merged for all models except SHT_UD. Using movies corresponding to Fig. 5, we found that the pattern shown in Fig. 5 is quasistationary on time scales of several milliseconds. Since we use a frame corotating with the pattern, this corresponds to the existence of an approximate helical Killing vector. In this sense, the remnant is not highly dynamic despite a strong nonlinear deformation.

Clearly, there is a relation between secondary vortices, hot spots, and density perturbation, although cause and effect are uncertain. As discussed for the irrotational model in [26], the hot spots apparently consist partially of hot matter trapped inside the secondary vortices, but also of regions where the temperature is raised by adiabatic heating at a local compression of the fluid flow. To substantiate that statement, we sample temperature and specific entropy along fluid trajectories and plot them as a function of the $\phi$ coordinate in the frame corotating with the density pattern, as shown in Fig. 6 for the irrotational model. One can see that compared to the temperature, the specific entropy shows only small variations along the trajectories (note that any decrease has to be caused by numeric errors such as heat conduction by numeric dissipation or the errors in the trajectory tracing). It is also apparent that some trajectories stay in the vortices, others pass through, and some stay a few cycles before leaving. The trajectories passing through
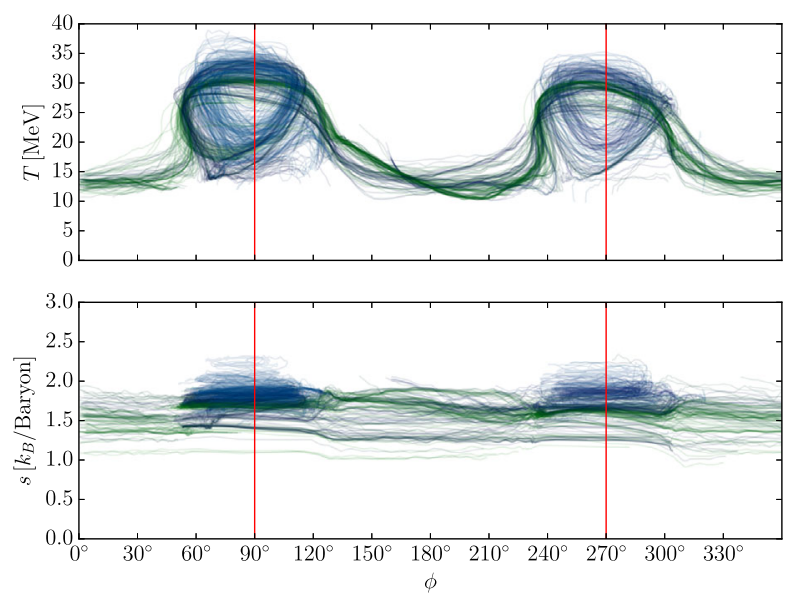

FIG. 6. Temperature (top panel) and specific entropy (bottom panel) along fluid trajectories as a function of the $\phi$ coordinate in the frame corotating with the density perturbation, for model SHT_IRR, in the time interval 5-11 ms after merger. Shown are only trajectories related to the hot spots, selected by the requirement that the maximum temperature is at least $30 \mathrm{MeV}$ and that the minimum density is above the bulk density. The monotonicity of $\phi$, defined as $\mu=\left(\int \dot{\phi}\right) /\left(\int|\dot{\phi}|\right)$, is denoted by colors ranging from green for $\mu=-1$ to blue for $\mu=0$.

show a strong adiabatic temperature increase in the regions rotated $\pm 90^{\circ}$ to the density pattern, and the material trapped in the hot spots has on average a somewhat larger specific entropy. Likely, both the thermal pressure and the varying centrifugal forces in the secondary vortices influence the density perturbation. The density perturbation, in turn, limits the possible fluid flows for a quasistationary pattern.

Our findings indicate that the early postmerger phase is not highly dynamic, but nevertheless very complex. It is doubtful whether it can be described correctly by linear mode analysis of axisymmetric stationary background models. This should be kept in mind when interpreting studies that use normal mode terminology to describe merger remnant oscillations, e.g. $[19,65,66]$ to name just a few (in fact [65] already cautions about the lack of a complete linear normal-mode analysis of merger remnants). In particular, interpreting the main frequency peak from the postmerger signal as the frequency of a linear $l=m=2 f$ mode seems questionable. A possible alternative would be to use the assumption of a helical Killing vector to compute strongly nonaxisymmetric systems. Modeling this phase correctly is important for the interpretation of future GW detections since only the early postmerger phase might be detectable, given the decaying amplitude.

Figure 5 shows a strong influence of the spin on the early remnant. Both the size and the shape of the secondary vortices differ, and so does the density perturbation. For the mixed spin case SHT_UD, the two main vortices merge later than for the other cases, and are also asymmetric. Moreover, there is only one secondary vortex. Finally, we 


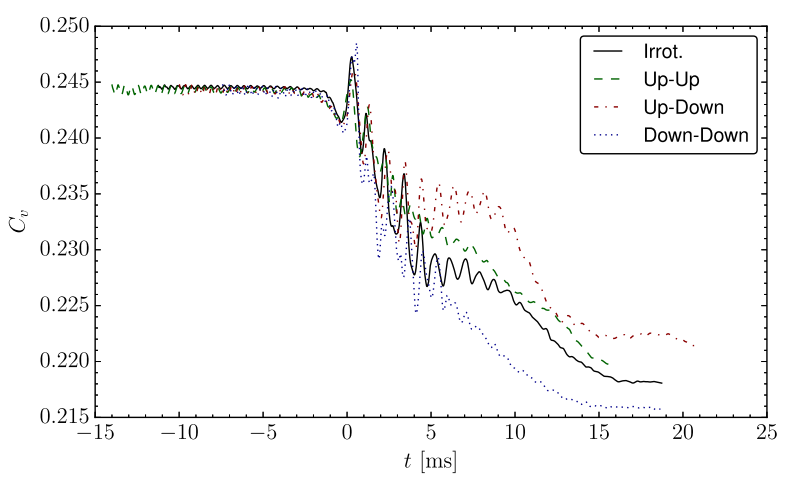

FIG. 7. Evolution of bulk compactness for different initial spins. The time $t$ is relative to the time of merger.

notice that the secondary vortices seem to be involved in transporting matter out of the remnant into the disk (compare Fig. 17 in [26] and the related discussion). Also this aspect is influenced by the spin. Assuming that our findings also apply to hypermassive stars, this might be relevant in the context of short gamma ray bursts, where the disk mass after collapse to a $\mathrm{BH}$ is a key parameter.

The differences in the structure are also reflected in the compactness of the remnant. Figure 7 shows the evolution of the bulk compactness introduced in Sec. II B. There are significant differences in the postmerger phase, up to $5 \%$. Those persist on the time scale of our simulations; the value at time $14 \mathrm{~ms}$ after merger is given in Table II (throughout this work, time of merger refers to the retarded time of the first maximum of the GW strain amplitude).

\section{Late remnant structure}

We now discuss the structure of the remnant in a later evolution stage, around $14 \mathrm{~ms}$ after merger. As shown in Fig. 8, the system has become more axisymmetric, in particular the hot spots have become ring shaped. The secondary vortices are still present, but have become less

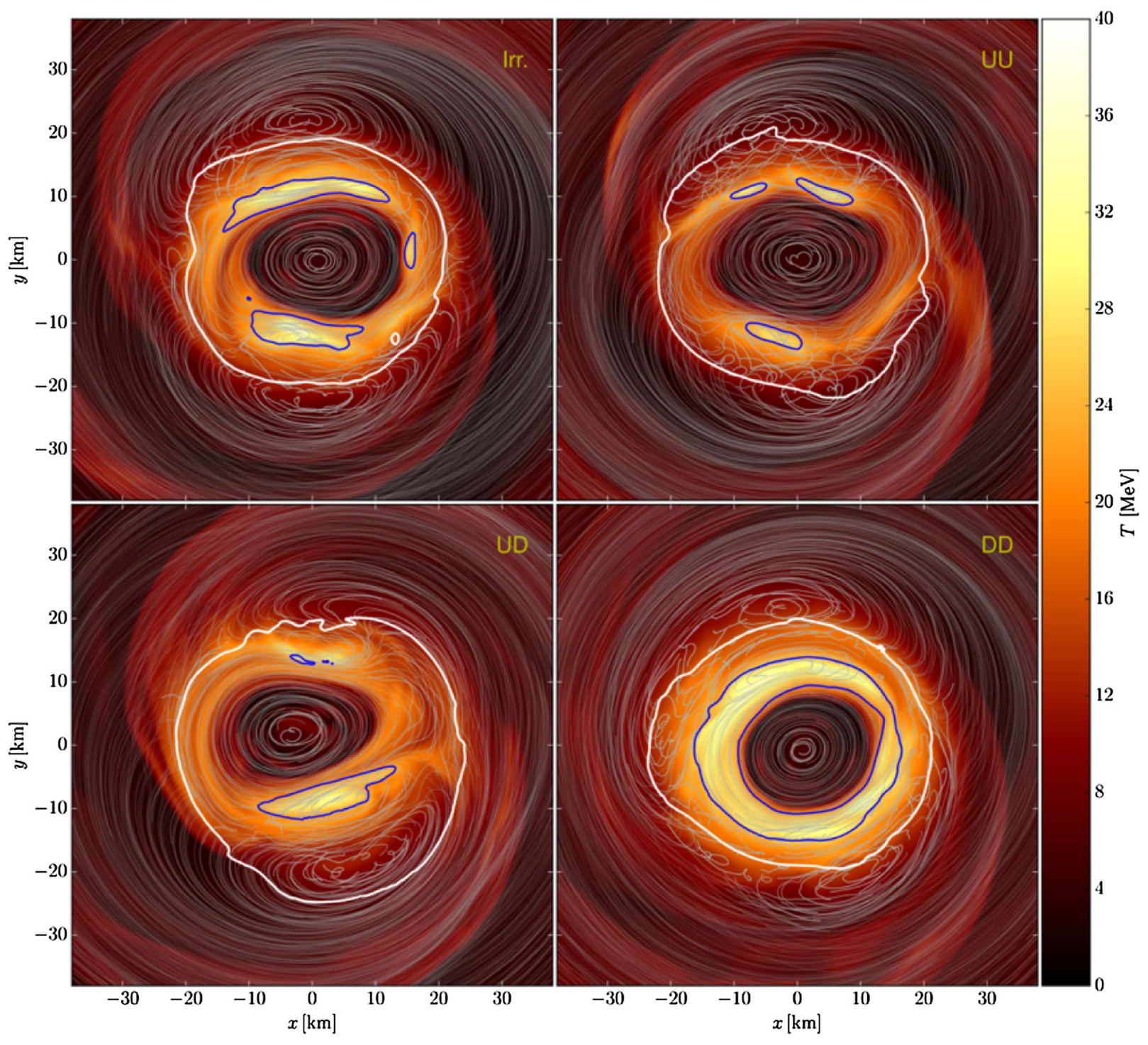

FIG. 8. Like Fig. 5, but showing the system $14 \mathrm{~ms}$ after merger. 

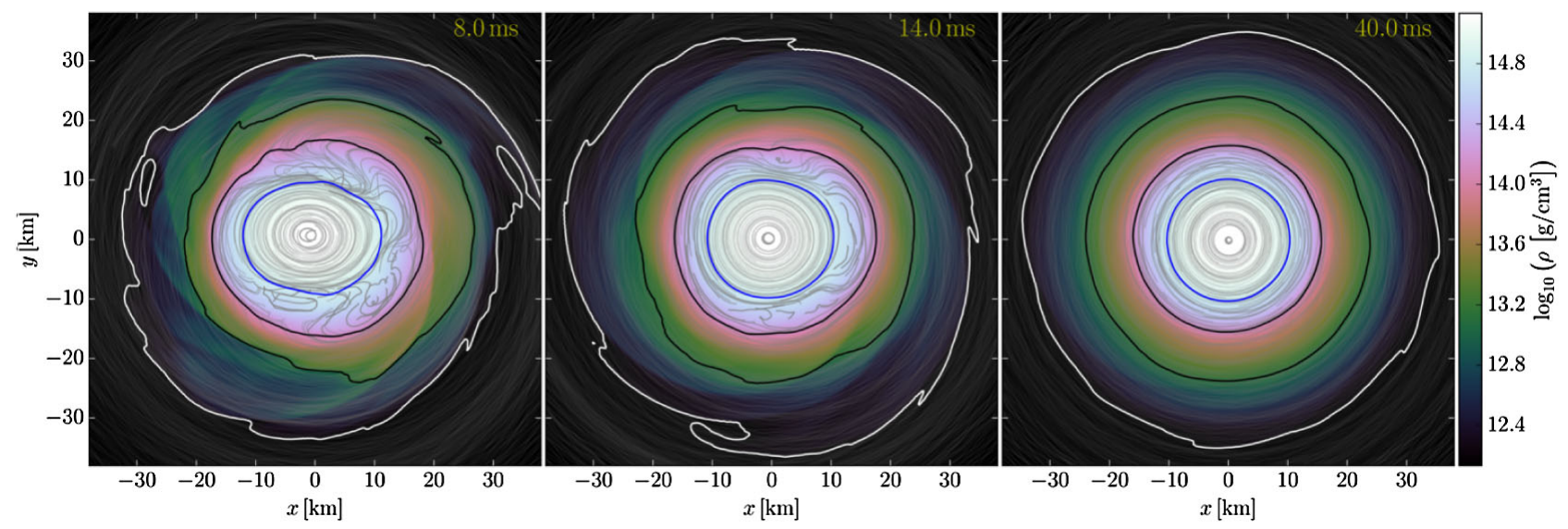

FIG. 9. Comparison of vortex structure in the equatorial plane for model APR4_EM at times 8,14 and 40 ms after merger. The density is shown as a color scale. The thick lines are isodensity contours $0.5,0.1,0.01,0.001$ of the maximum density. The thin grey lines are fluid trajectories in the frame corotating with the $m=2$ density perturbation, in a time window $\pm 1 \mathrm{~ms}$ around the snapshot.

localized. This might be a consequence of the reduced density perturbation, since the early vortices are situated in the wakes of the most radially extended parts. Note that the mixed spin model SHT_UD is still very asymmetric, but the pattern changed drastically with respect to the earlier state shown in Fig. 5. The system underwent a rearrangement of vortices within 2-3 ms. In contrast, the changes of the other models took place more gradually.

To rule out that the remnant structure described so far is specific to the corner case of stable remnants of equal-mass binaries, we also investigate the vortex structure for the two supramassive models, APR4_EM and APR4_UM, one of which is an unequal mass model. Figures 9 and 10 show the trajectories on top of the mass density (the temperature was not available for those runs). Again, we find secondary vortices phase locked with the main density perturbation, although they are less pronounced for the equal mass model. Not surprisingly, the unequal mass model has a strongly asymmetric vortex structure. To a lesser degree, also the equal mass system deviates from $\pi$-symmetry. The asymmetry correlates with the vortices and might be caused by their dynamics in the early postmerger phase. In any case, the system becomes more axisymmetric with time, which rules out an unstable $m=1$ mode as the cause.

In contrast to earlier stages, the remnants at the time shown in Fig. 8 can be described roughly as differentially rotating objects, albeit still strongly nonaxisymmetric and with superposed vortices. It therefore becomes meaningful to measure the rotation profile. To this end, we use two methods. First, we compute the angular velocity averaged
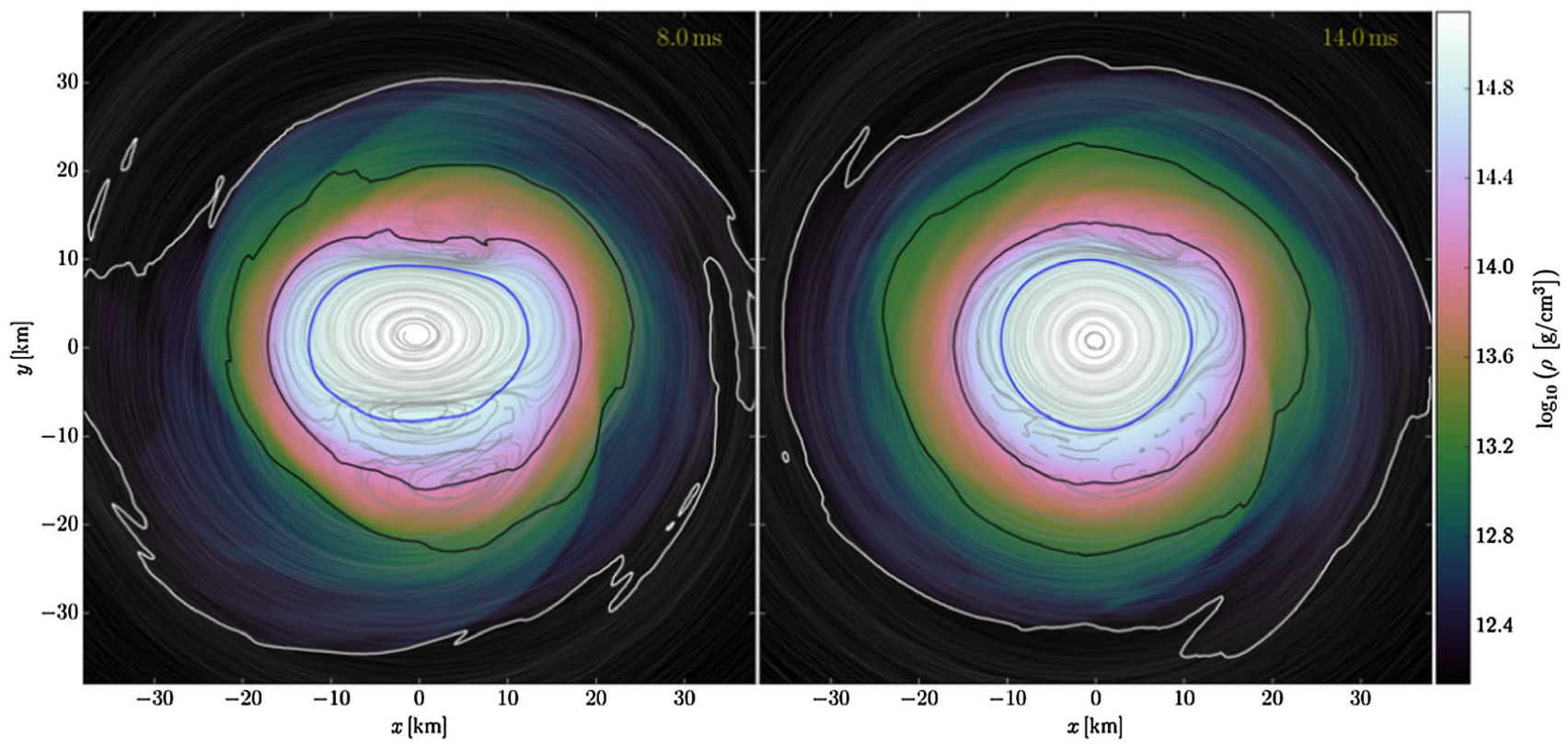

FIG. 10. Like Fig. 9, but showing model APR4_UM at times 8 and 14 ms after merger. 
in the $\phi$ direction at fixed radii. Second, we average the angular velocity and the radial coordinate of fluid elements along their trajectories over a fixed time interval.

The results are shown in Fig. 11. Not surprisingly, the trajectories making up the secondary vortices show a large spread. On average, however, the angular velocity of those trajectories coincides both with the maximum average in the $\phi$ direction and with the angular velocity of the density perturbation (shown as horizontal lines in Fig. 11). Apparently, fluid elements cannot overtake the elevated parts of the deformation pattern. Since the density deformation is responsible for the GW signal, this explains our observation that the maximum rotation rate is approximately given by half the GW frequency. As shown in Table II, the difference between maximum rotation at $14 \mathrm{~ms}$ after merger and the main postmerger peak in the GW spectrum is between $0.6 \%$ and $14 \%$ for our models. Since the frequencies are evolving, it is more meaningful to compare the time-dependent maximum rotation rate to half the instantaneous GW phase velocity (cf. Fig. 15 in [39]). For this, we considered times $>5 \mathrm{~ms}$ after merger, except for the up-down model, where the maximum rotation rate around the origin is still the central one until $\approx 10 \mathrm{~ms}$ after merger (due to the off-center vortex, see Fig. 8). We also ignored times after $25 \mathrm{~ms}$ for the APR4_EM model because of the low GW amplitude. On those intervals, the $L_{1}$-norm of the difference is $\lesssim 6 \%$ for all models. The relation between maximum rotation rate and GW frequency was also observed in $[9,25,27]$. We also find that the remnant cores rotate slowly. The same was also found in $[9,25,27]$ for various hypermassive and supermassive remnants with different EOSs, and in [26] for our irrotational model. Note that the main vortex for the mixed-spin model is displaced by a secondary vortex (see Fig. 8), which explains the trajectories with high angular velocity at $r<5 \mathrm{~km}$ visible in Fig. 11. As for unequal mass models, the central rotation rate is not meaningful in this case since the center is not the center of rotation.

To estimate the error of the rotation rates, we use the maximum rotation rates reported in [39]. Although the resolution test in there was done for a magnetized version, the influence of the magnetic field on the rotation profile was shown to be small. Under the optimistic assumption of first order convergence starting at the standard resolution, we estimate the error of the rotation rates around $3 \%$. The impact of the initial spin on the maximum rotation rate

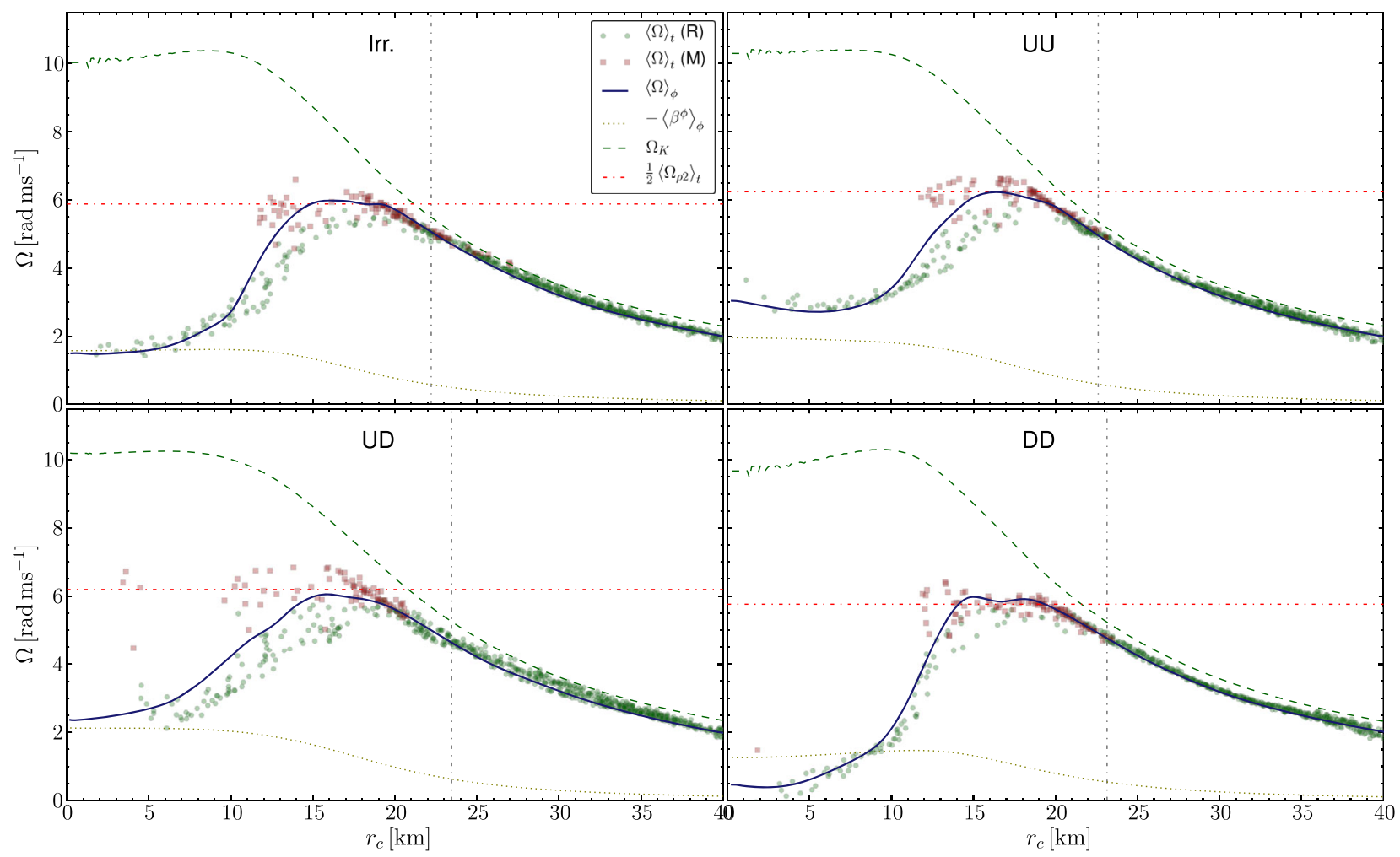

FIG. 11. Angular velocity in the equatorial plane as observed from infinity at $14 \mathrm{~ms}$ after merger, averaged over a time interval $\pm 1 \mathrm{~ms}$. The solid line shows the $\phi$ average of angular velocity. The markers are the time average of angular velocity and circumferential radius along traced fluid trajectories. Trajectories of mixed prograde and retrograde nature (with respect to the corotating frame) are marked by red squares, the others by green circles. The vertical lines mark the radius at which the average density is 5\% of the central one, and the horizontal lines the angular velocity of the $m=2$ component of the density perturbation in the equatorial plane. The dashed curve is the estimated orbital angular velocity of a test mass in corotating orbit. The dotted curve is the $\phi$-averaged shift vector component $\beta^{\phi}$ as an estimate for the frame dragging coefficient. 
STRUCTURE OF STABLE BINARY NEUTRON STAR ...

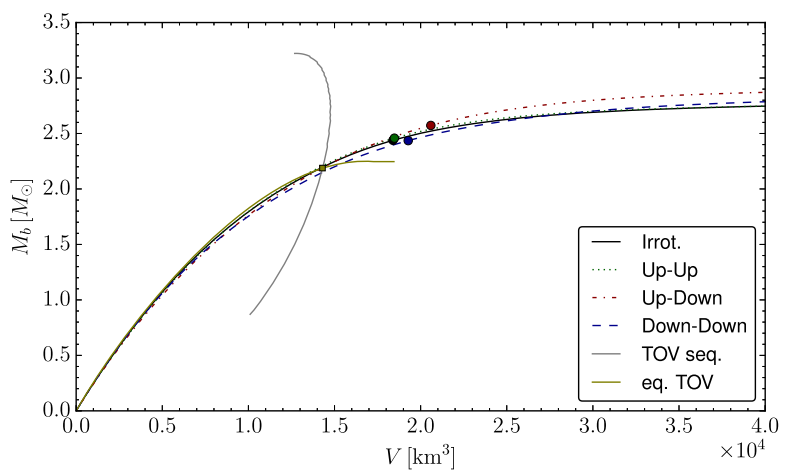

FIG. 12. Baryonic mass versus proper volume contained in isodensity surfaces, at $14 \mathrm{~ms}$ after merger. The symbols mark the "bulk" values. We also show the relation of bulk mass versus bulk volume for a sequence of TOV stars with the same EOS as the initial data. The intersection with the remnant profiles defines their "TOV core equivalents." The profile of the core equivalent shown in the plot corresponds to model SHT_IRR.

reported in Table II is only around 2\%. The amount of differential rotation shown in Fig. 11 on the other hand exhibits larger differences, exceeding the numerical error. We note there is a possibility that the differential rotation might in reality be reduced quickly by viscous effects induced by strong magnetic fields on small length scales. Those can be generated in principle by $\mathrm{KH}$ and magnetorotational instabilities that are not resolved in numerical simulations with standard resolutions (see [63]). The possible effect of such viscous damping was recently pointed out by [67] using a simple alpha-viscosity model.

We now discuss the radial mass distribution of the remnants. Figure 12 depicts the mass-volume relations introduced in Sec. II B for all spin configurations. We observe differences in the core around a few percent between the different spin configurations, comparable to the magnitude expected from the bulk compactness shown in Fig. 7. In [26], we found that the core of the remnant for irrotational model has a mass profile very similar to that of a TOV solution. The mass-volume relation for this core-equivalent TOV model is shown as well in Fig. 12. In addition, Fig. 13 shows the same data in terms of density versus the volumetric radius of the corresponding isodensity surface. We find that the radial mass distributions of remnant and TOV core equivalent agree indeed well. Centrifugal forces become important for volumetric radii $>14 \mathrm{~km}$ (when comparing to Figs. 5 and 8 note that they use the circumferential radius instead). This agrees with the rotation profiles which show that the matter approaches Kepler velocity in those outer layers.

\section{Gravitational waves}

For all our simulations, we extracted the GW signal using the methods detailed in Sec. II C. In order to reduce boundary effects when computing Fourier spectra, we first applied the tapering window function described in [68],
PHYSICAL REVIEW D 96, 043019 (2017)

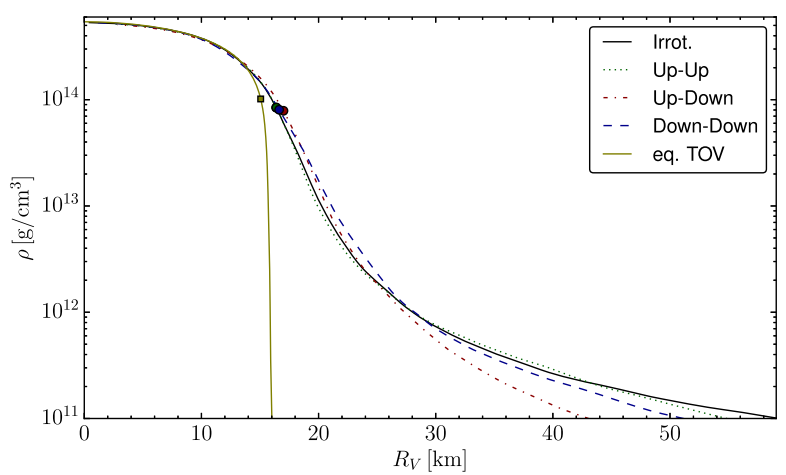

FIG. 13. Density versus volumetric radius for isodensity surfaces at $14 \mathrm{~ms}$ after merger. The symbols mark the bulk values. For comparison, we also show the TOV solution approximating the core (see Fig. 12).

with a tapering duration of $2 \mathrm{~ms}$. The results are shown in Figs. 14-19 (the strain is also publicly available in the Supplemental Material of this article [64]). The waveforms look quite typical, in particular the short minima after merger are fairly common for BNS mergers. Using the methods described in Sec. II C, we have shown that those can be described as overmodulation, i.e. a zero crossing of the signal amplitude. For all models, we detected phase jumps at the location of the first two minima, except model APR_EM where only the first minima was identified as phase jump. The jumps are particularly visible in the phase velocity, which exhibits strong peaks that are not present in the jump-corrected phase.

The presence of a phase jump shortly after merger makes it more difficult to interpret the spectra. In particular timefrequency maps will exhibit a decrease in amplitude near the phase jump since the signal before and after partially cancels. This might in principle be relevant for any classification scheme of GW merger events (such as $[74,75])$, or construction of matched filtering templates (such as [76]), if they are carried out in the frequency or time-frequency domain. However, the improvement of GW analysis pipelines is beyond the scope of the current paper. Here we only try to estimate the potential impact of cancellation effects on the Fourier spectrum. For this, we compute also the spectrum of a modified signal given by $h e^{-i \delta \phi}$, where $\delta \phi$ is the jump correction introduced in Sec. II D. We stress that the factor $e^{-i \delta \phi}$ affects the frequency only in short intervals around the jumps. We therefore attribute any large changes in the spectrum to cancellation effects between the segments separated by the jumps.

As shown in Figs. 14 to 19, the impact is dramatic. Specifically, all spectra of the standard strain show a broad peak between the frequency ranges of inspiral and postmerger phase, separated from the main postmerger peak by a relatively steep valley (e.g. at $1.75 \mathrm{kHz}$ in Fig. 14). The location of the low-frequency peak and the valley change significantly. The effects of the phase jumps are also noteworthy since low-frequency peaks have been 

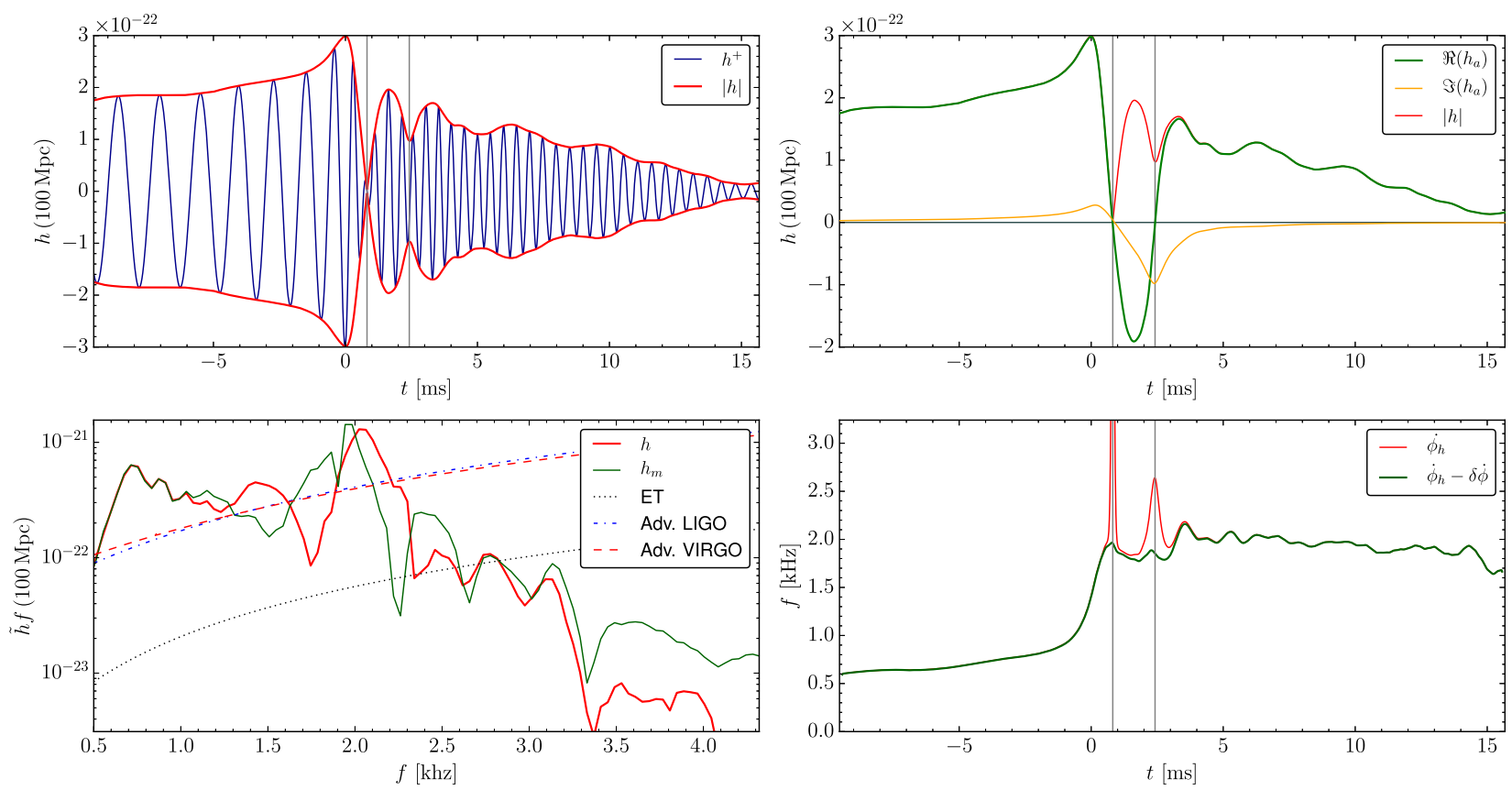

FIG. 14. GW signal for model SHT IRR. Upper left panel: $l=m=2$ component of the GW strain. Upper right: The evolution of the complex amplitude $h_{a}$ defined in Sec. II D, indicating two phase jumps after merger (marked by the vertical lines). Lower right: Phase velocity both for the continuous phase $\phi_{h}$ of $h$ as well as the phase $\phi_{h}-\delta \phi$ corrected for phase jumps. Lower left: Fourier spectrum of $h$ at $100 \mathrm{Mpc}$ compared to GW detector design sensitivity curves [advanced LIGO, zero detuning, high power [69], advanced Virgo [70,71], Einstein Telescope (configuration ET-B) [72,73]]. For comparison, we also show the spectrum of the strain after removing the phase jump, given by $h_{m}=h e^{-i \delta \phi}$.

interpreted as combination frequencies of $m=0$ and $m=$ 2 oscillations of the remnant in [65], while [77] correlated those peaks phenomenologically with the compactness if the initial NSs. Our findings indicate that, at least for the cases at hand, those peaks might not be the result of one or several physical oscillations, but could be generated by the superposition of a phase-cancellation induced valley onto an underlying frequency distribution that is much
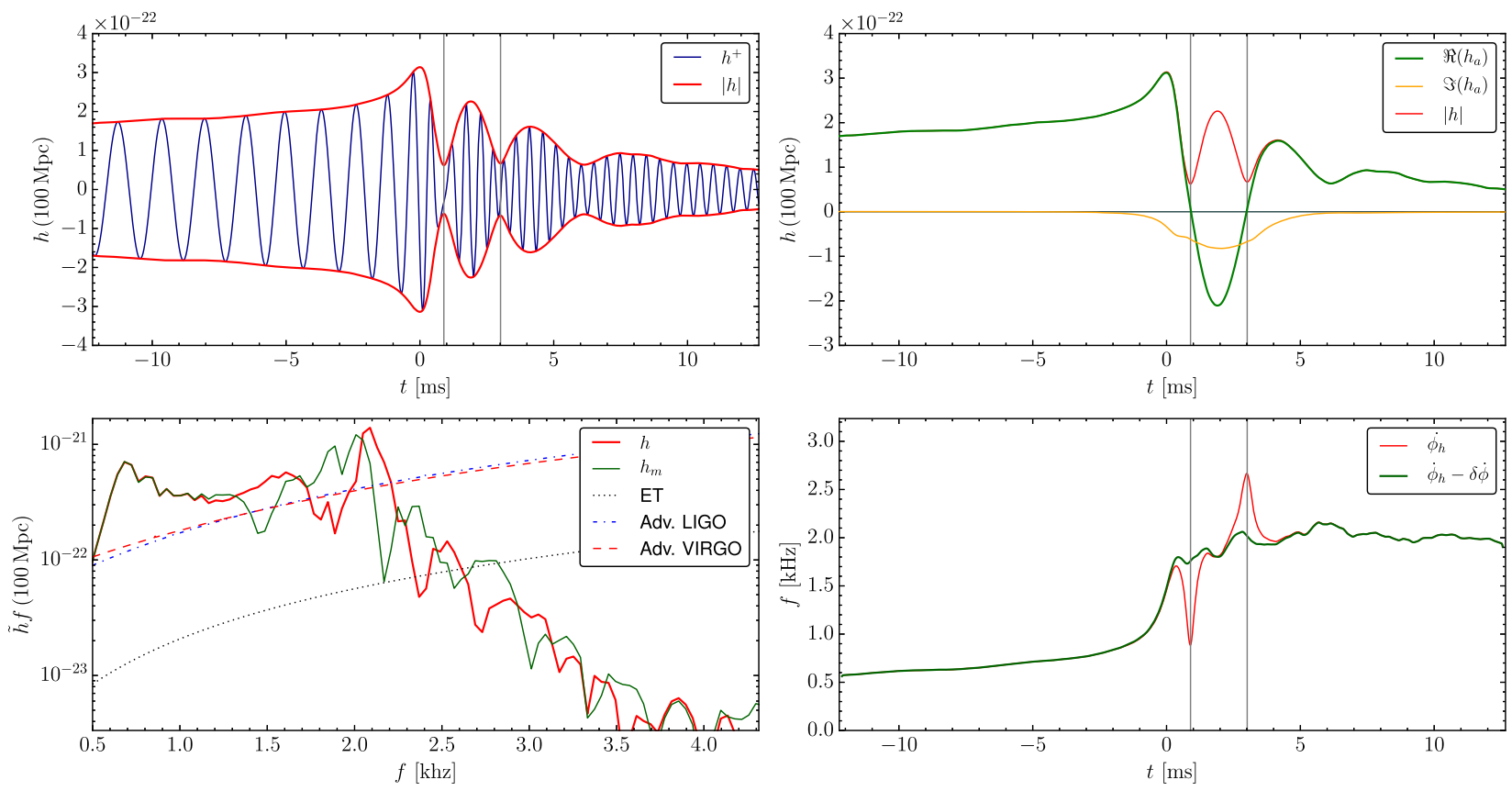

FIG. 15. Like Fig. 14, but for model SHT_UU. 

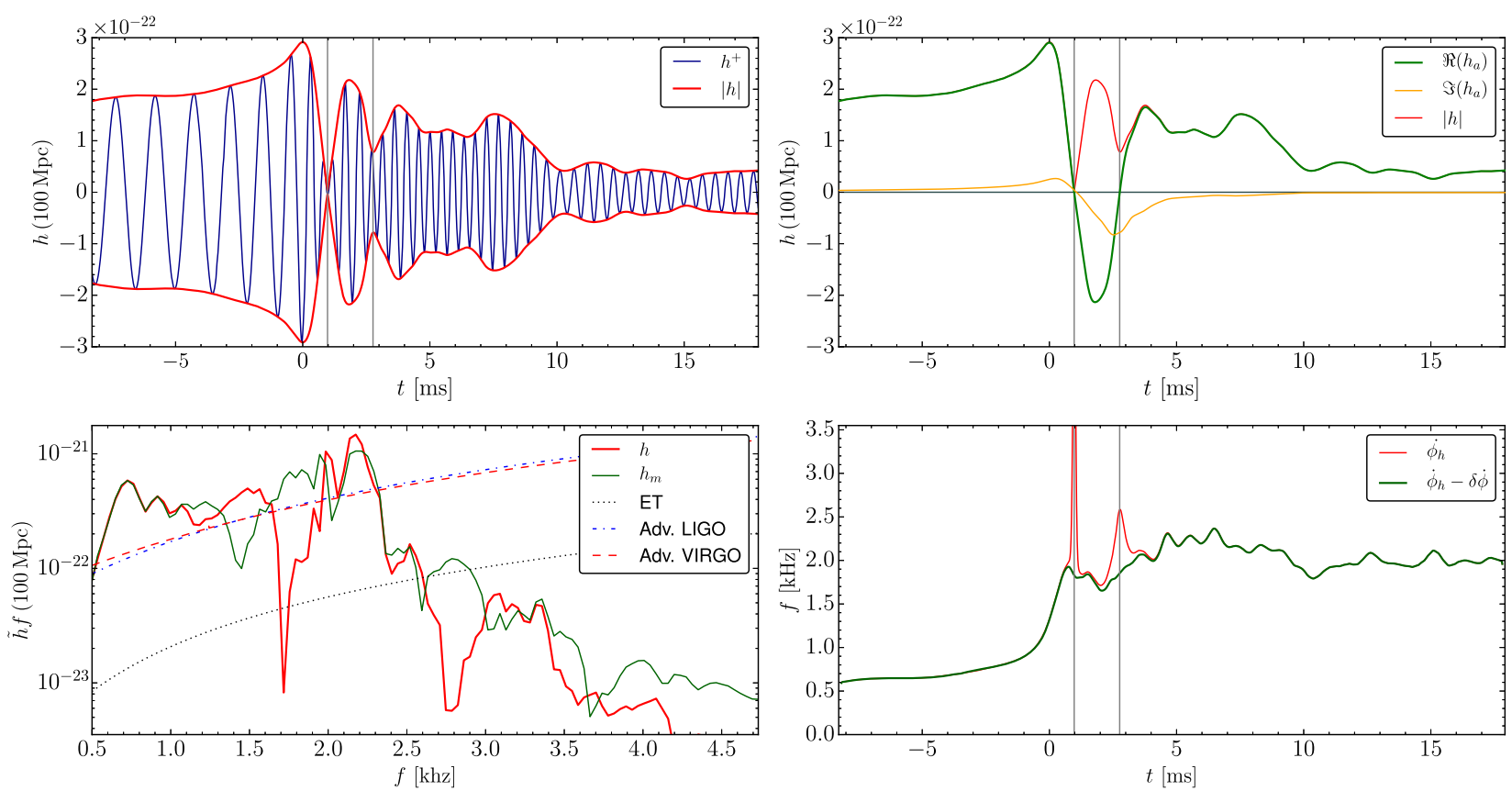

FIG. 16. Like Fig. 14, but for model SHT_UD.

smoother. Although this possibility complicates GW data analysis, it also gives reason to hope that the behavior of the actual frequency evolution of merger events is less complex than suggested by the strain, once phase jumps are accounted for.

Now that we have established the presence of overmodulation in GW signals, we propose a model to explain the physical causes. For this, we consider the dynamics of the merger in a rotating frame with angular velocity
$\Omega=\dot{\phi} / 2$, where $\phi$ is the jump corrected and smoothed phase of the $m=2$ component of the GW strain. The exact rotation rate is not important, only that it is slowly varying and in phase with the GW signal on average. We then consider the $m=2$ multipole moment $Q_{c}$ in the corotating frame. The multipole moment $Q_{I}$ in the inertial frame is given by $Q_{I}=Q_{c} e^{i \phi}$. We now regard $Q_{c}$ as a slowly varying complex-valued amplitude modulating the oscillation given by the factor $e^{i \phi}$. Of course, $Q_{c}$ can undergo a sign change.
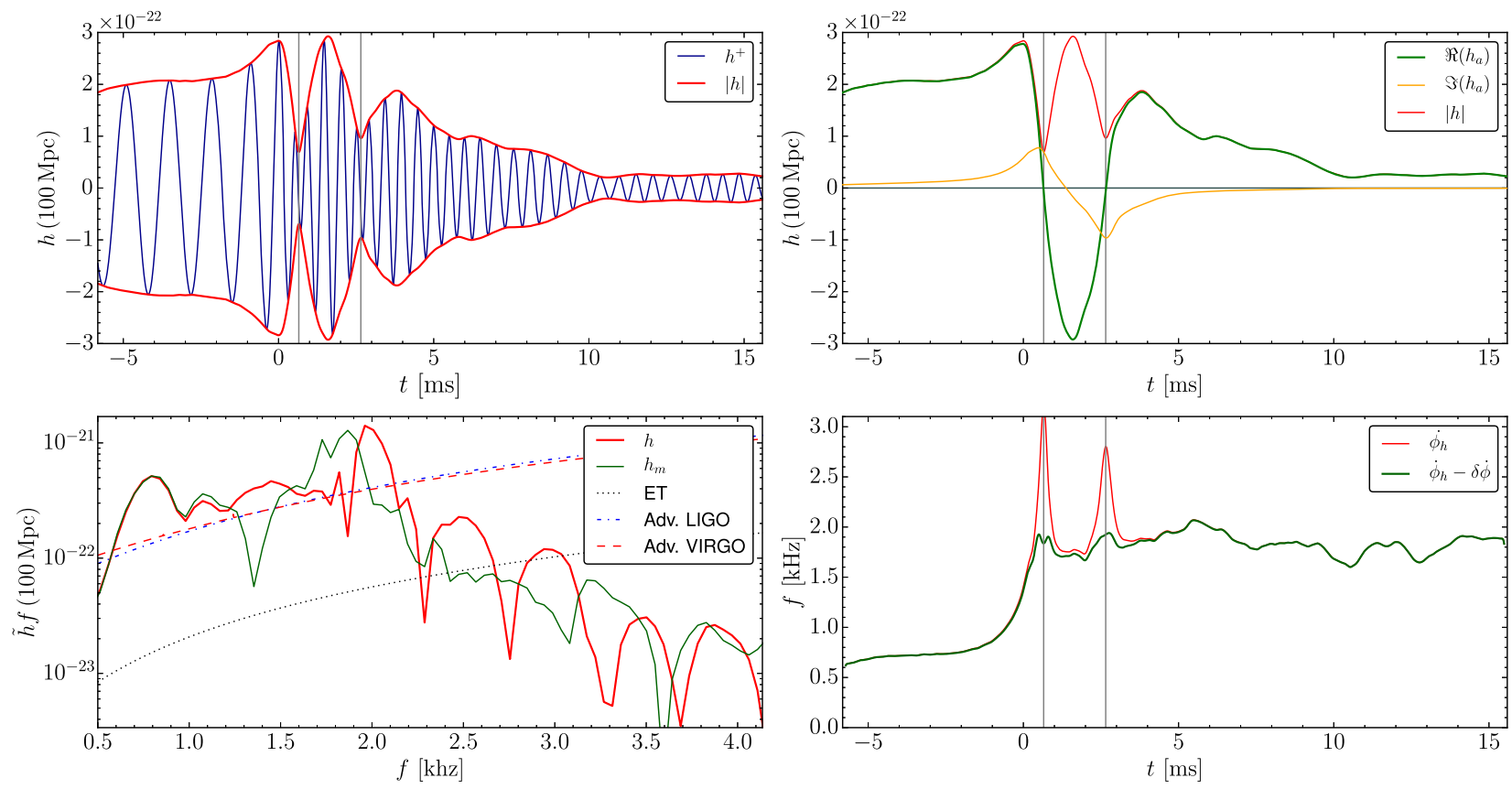

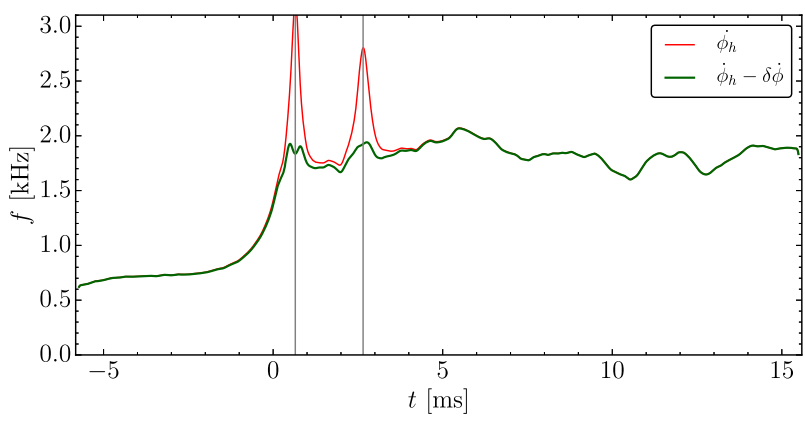

FIG. 17. Like Fig. 14, but for model SHT_DD. 

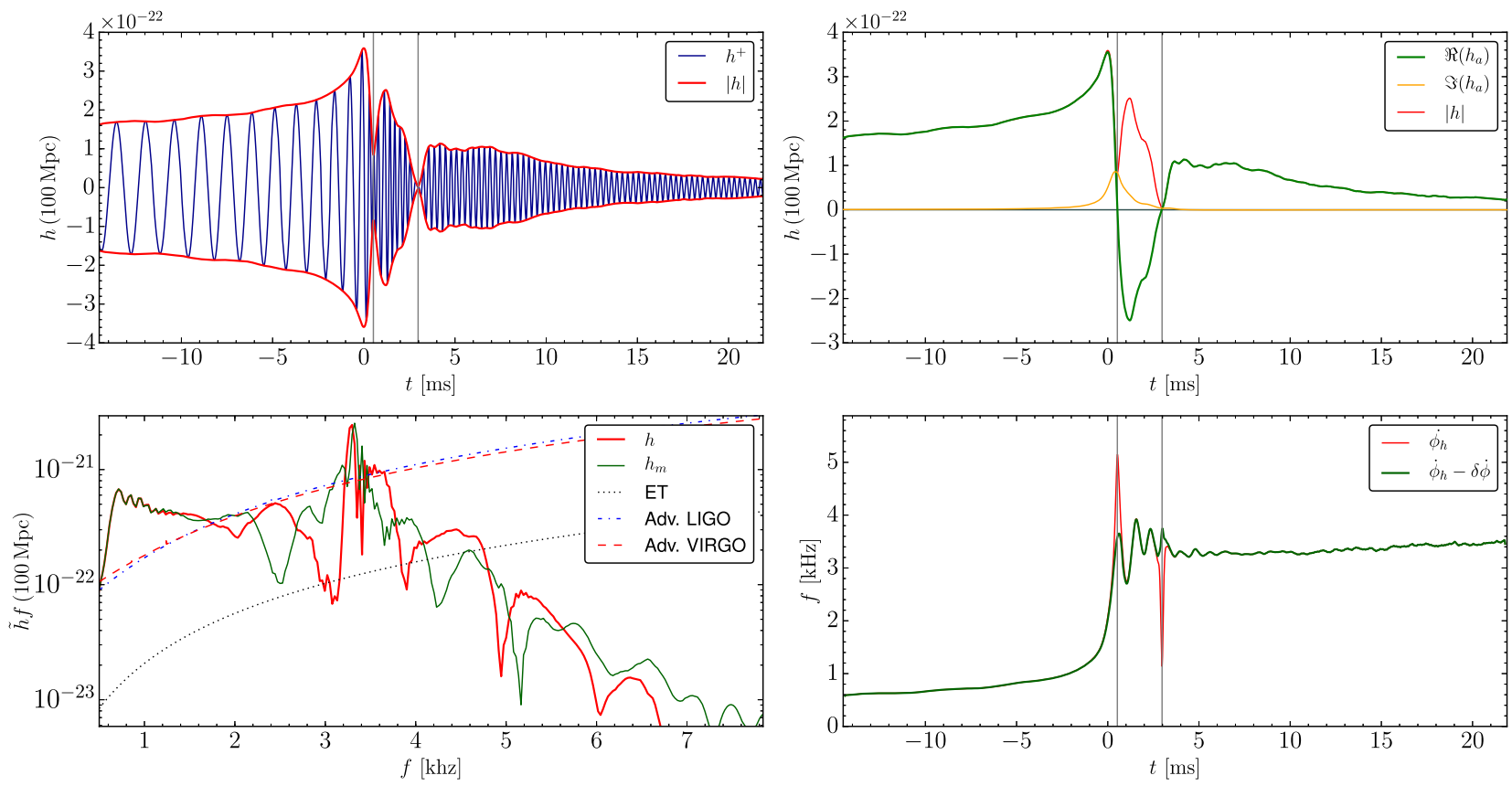

FIG. 18. Like Fig. 14, but for model APR4_UM.

For the $m=2$ moment, a sign change means that the principal axes are interchanged. It is plausible that this happens during merger. We therefore have all the ingredients for overmodulation of $Q_{I}$. The only complication is that the GW strain is given by the second derivative of $Q_{I}$. For the strain $h=h_{c} e^{i \phi}$, we thus obtain

$$
h_{c}=\ddot{Q}_{c}+2 i \omega \dot{Q}_{c}+\left(i \dot{\omega}-\omega^{2}\right) Q_{c}
$$
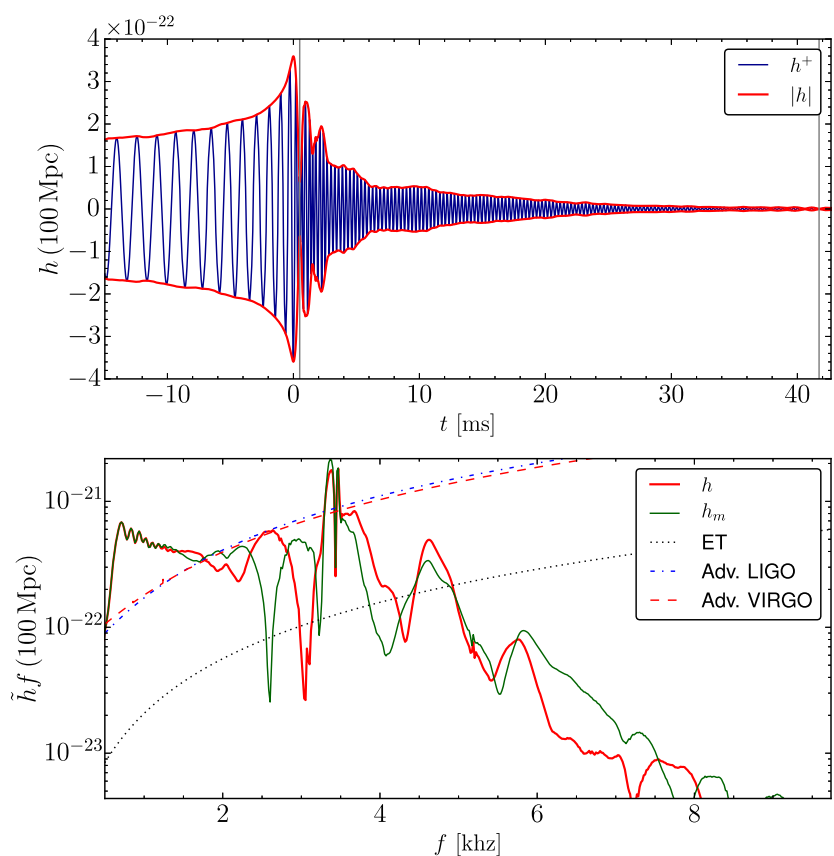

where $\omega=\dot{\phi}$. For the simplest case of a linear zero crossing of the form $Q_{c}=\dot{Q}_{c}\left(t-t_{j}\right)$, and assuming $\dot{\omega} \approx 0$, we obtain $h_{c}=i \omega \dot{Q}_{c}\left(2+i \omega\left(t-t_{j}\right)\right)$. This is exactly the form assumed for the jump fitting shown in Sec. II D, with $k=\omega / 2$. For this simple case, we find that the strain itself does not exhibit a zero crossing, but assumes a minimum determined by the speed of the zero crossings of $Q_{c}$. However, the strain does undergo a phase shift by $\pi$.
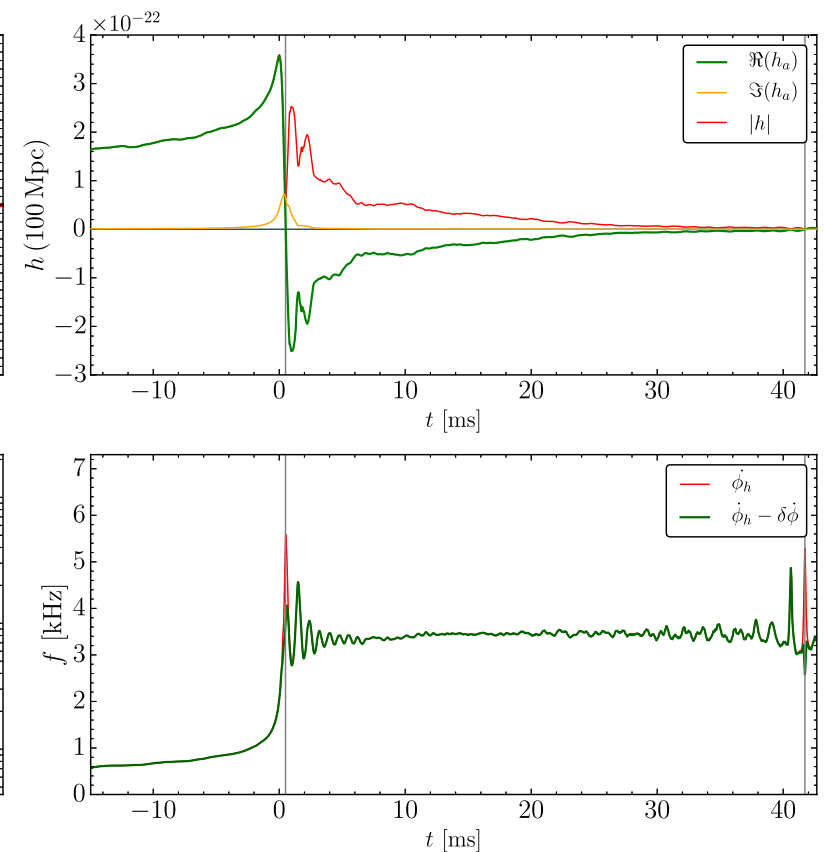

FIG. 19. Like Fig. 14, but for model APR4_EM. Note the instantaneous frequency variations after $30 \mathrm{~ms}$ are artifacts of the low signal amplitude. 
This simple model can also explain the large second derivative of strain amplitudes often seen in gravitational waveforms, without requiring a sudden change of the multipole moment in the rotating frame. We caution that some of the broader peaks in phase velocity might have different causes than near zero crossing of $Q_{c}$. For the sharp peaks, however, the frequency changes by more than $1 \mathrm{kHz}$ within less than $0.5 \mathrm{~ms}$. It is implausible that the remnant's oscillation frequency or rotation rate changes so drastically within a period shorter than the dynamical time scale; our model provides a more natural explanation.

For example, in [9] we observed some strong peaks in the GW phase velocity during merger, which we interpreted as a consequence of a compactness maximum of the remnant. After reanalyzing the data with the new methodology, we find that the frequency peak at merger is partially due to a phase jump. Another example is the GW strain for model APR4_UM shown in Fig. 18, which has a very pronounced minimum around $3 \mathrm{~ms}$ after merger that we left unexplained in [25]. We now find that this minimum is a very clear case of a phase jump, judged by the sharp peak in the instantaneous frequency. Thus, the minimum is caused by a simple zero crossing of the quadrupole moment. The characteristic behavior of the phase makes other explanations very unlikely. In particular, we rule out that the minimum is the superposition of a damped mode excited at merger and another mode growing unstably or that it is a simple beating phenomenon between two modes. We are not aware of any GW data analysis targeting temporary minima. Nevertheless, if postmerger signals that turn off temporarily should be observed by GW astronomy, such considerations will become crucial for the physical interpretation.

After discussing the generic GW features, we now turn to the impact of the NS spin. Figures 20 and 21 show a comparison of the GW spectra of the four cases. Further, we report in Table II the frequency of the maximum of the power spectrum (excluding the part corresponding to inspiral) as well as the instantaneous frequency at merger time. We estimate the finite resolution error of the main postmerger frequency peak around $2 \%$, based on the resolution study in [39]. Although the location of the main peak is affected by the spin, the shift is generally comparable to the width of the peak. One noteworthy feature is the appearance of a small side peak for model SHT_UD. As seen in Fig. 16, this feature is not caused by cancellation effects related to phase jumps. By comparing to the instantaneous frequency (the phase velocity) shown in Figs. 20 and 21, we find that the splitting of the main peak is caused by a small frequency change occurring around $8 \mathrm{~ms}$ after merger. This coincides with a rearrangement of the vortex structure that can be seen in Figs. 5 and 8 . One plausible explanation would be that the rearrangement of the fluid flow slightly changes the moment of inertia, thus causing a small decrease in the rotation rate.
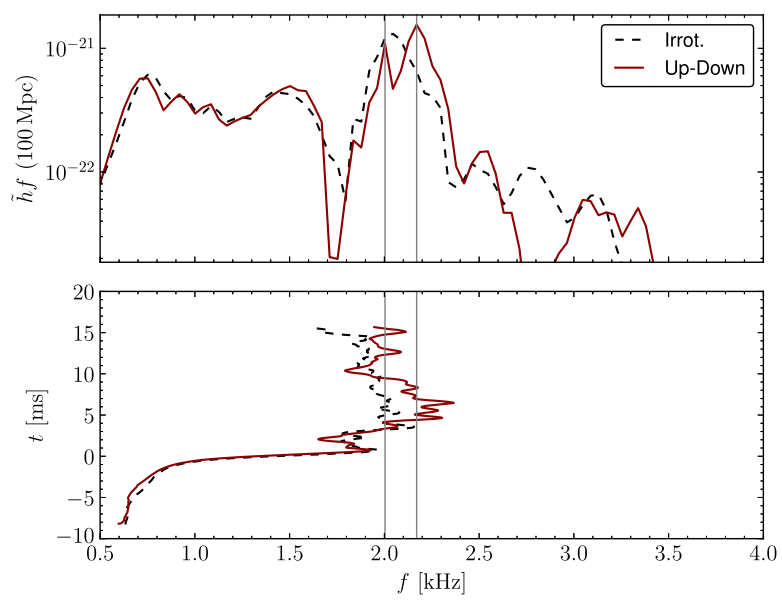

FIG. 20. Upper panel: GW power spectrum for irrotational and up-down spin configurations. Before Fourier analysis, the signals have been cut to the common time interval with respect to merger time to exclude differences due to simulation length. Lower panel: Time evolution of the jump-corrected instantaneous frequency ( $t=0$ is the time of merger). The vertical lines mark the peaks of the power spectrum for the up-down case.

Note that also the fluid flows of other models undergo changes. The difference between the up-down and the irrotational model is that the transition happens more smoothly for the latter, and correspondingly the instantaneous frequency shows a more continuous drift as well. This indicates that drifts of the frequency in the late postmerger phase might be caused by a slow change of the remnant's vortex distribution, offering an alternative explanation to the common notion that the angular momentum carried away by GWs is responsible. We recall that angular momentum loss affects the frequency not simply by a change in rotation rate, but mostly by the change in compactness (see also the discussions in $[11,19,78])$, which makes it difficult to
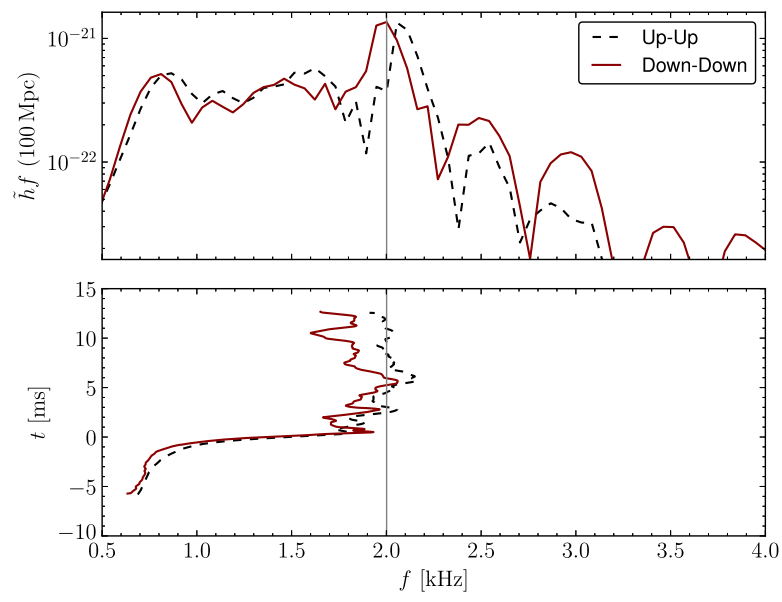

FIG. 21. Like Fig. 20, but comparing the up-up and down-down spin configurations. The vertical line marks the peak in the downdown spectrum. 
distinguish from the effects of internal structural rearrangements. For the gradually changing models, one could argue that the angular momentum loss is a common cause both for changes of vortex distribution and GW frequency; the more rapid change observed for the up-down model seems to indicate otherwise, however.

A rearrangement of vortices might also lead to strong strain amplitude variations in some cases, which might explain minima sometimes observed in numerical waveforms. In [34], it was suggested that the rise of the GW amplitude following minima in specific cases might be caused by the growth of unstable modes. Evidence for such instabilities has only been found for hypermassive neutron star (HMNS) models with rapidly rotating core however (see e.g. $[79,80])$. The reduction in amplitude around 8 to $10 \mathrm{~ms}$ after merger shown in Fig. 16 provides at least one example where the change can be attributed to a change of the vortex structure that takes place simultaneously (as can be seen in a movie available in the Supplemental Material [64]). There is no reason to assume such a rearrangement could not produce a temporary minimum instead; in fact, we observed a minima coinciding with a vortex rearrangement in [39] (for the unequal mass $\mathrm{H} 4$ model). In this context, we also note that some of the waveforms presented in [30] (e.g. models ALF-120150, H4-120150) show the sharp maxima or minima in the instantaneous frequency characteristic for phase jumps exactly at the location of strain minima. The relation between vortex structure and strain amplitude clearly deserves further study.

\section{E. Disk and matter ejection}

We now turn to the effect of the spin on the disk and the ejected matter. To this end, we first compute the total mass outside a coordinate sphere with proper volumetric radius of $26 \mathrm{~km}$, defining a tentatively named "disk mass." We stress that the mass depends strongly on the cutoff radius. Our choice of the latter was motivated by Fig. 16 in [26], showing the isodensity contours for our irrotational SHT model in the meridional plane (for comparison, the cutoff radius corresponds to $20 \mathrm{~km}$ in the simulation coordinates used in the figure). Further, the bulk surfaces shown in Fig. 8 are completely contained within a coordinate sphere with proper volumetric radius $24 \mathrm{~km}$.

Not all of this matter really forms a disk. The parts at large radius typically have strongly eccentric orbits with periods larger than the evolution time. This matter will eventually fall back onto the inner system and might give rise to interesting phenomena outside the scope of this paper. Of course, the transition between circularized disk and eccentric fallback component is gradual. To give a number however, we computed the matter outside a radius of $60 \mathrm{~km}$, as in [25]. The masses outside 26 and $60 \mathrm{~km}$ are reported in Table II for the SHT models. We find a considerable impact of the spin on both measures. For the models at hand, the disk mass for the up-down (down-down) case is only 55\% (80\%) of the mass for the up-up case (or the almost identical irrotational case). Although we have no convergence test, we are optimistic that those differences are larger than the numerical error. The inner parts of the disk are also visible in Fig. 13, showing density versus volumetric radius of the corresponding isodensity surface. Here we find that the density falls off most strongly for model SHT_UD.

In this context it is worth noting that the matter in the disk is not formed completely during merger, but material is migrating outwards continuously. In [26] we have shown for the irrotational model that many fluid trajectories ending up in the disk interact with secondary vortices at some point. Since the latter are sensitive to the spin, the impact of the spin on the disk mass is not necessarily caused only by the different centrifugal forces during the merger. Lacking a clear measure, we did however not distinguish the amount of matter migrating to the disk immediately from the amount thrown out later.

In order to estimate the amount of ejected matter, we use the same tools as in [25] (see also the discussion in [9]). In short, we compute the time integrated flux of unbound matter according to the geodesic criterion through several spherical surfaces. As explained in [25], we use the maximum obtained from the different surfaces $(r=111$, $148,222,295,443,591,916 \mathrm{~km}$ for the SHT models) as a best estimate. The results are given in Table II. For a more direct comparison of results for the different spins, we also report the ejected mass measured using the same extraction sphere at fixed radius $r=222 \mathrm{~km}$. We find that all SHT models eject only small amounts of matter $\lesssim 10^{-3} M_{\odot}$. There are however strong differences between the different spin configurations. Most matter is ejected for the up-up case, and least for the irrotational and the mixed cases. We note that this is opposite to the relation found in [9], for a model with the SHT EOS, but in the lower hypermassive mass range. In this case, the irrotational model ejected more matter than the up-up model. This was connected to the ejection mechanism: the strong radial oscillations of the remnant launched many waves that kicked matter out of the disk, and the strength of the oscillations was greater for the irrotational case.

Note the accuracy of the ejected mass is difficult to estimate. There are several sources of error: the validity of the geodesic assumption, the finite resolution, and interaction with the artificial atmosphere. For the resolution error, we again use the resolution studies for magnetized versions of the APR4 models in [25,39], which provided estimates around $17 \%$ and $50 \%$ for the equal and unequal mass models, respectively. Lacking a dedicated convergence test, we assume a finite resolution error around 50\% for the SHT models as well. Regarding the artificial atmosphere, we should note that the total baryon mass of the system increases by up to $10^{-3} M_{b}^{\text {tot }}$ (i.e. more than 
the ejected mass) during the SHT EOS simulations. However, this number refers to the whole computational domain and cannot be added to the error of the ejecta mass. The interaction of ejected matter with the artificial atmosphere actually decreases the unbound mass. This was found by monitoring the radial distribution of ejected matter using histograms and is also indicated by an animation for model SHT_UU showing that the matter becomes bound again at a large radius while expanding into the artificial atmosphere. Using the same animation (available in the Supplemental Material [64]) we also verified that the unbound matter traverses refinement boundaries without visible artifacts. All in all, we roughly estimate the mass to be accurate within a factor of 2 , unless the ejecta mass is below $10^{-4} M_{\odot}$, in which case we only provide an upper limit.

To identify the ejection mechanism for the up-up model of the present work, we produced movies visualizing unbound matter together with density or temperature in the meridional and equatorial planes. We found that, for this model, almost all matter is ejected in a single wave consisting of two concentric rings above and below the orbital plane. Those animations also gave the impression that the matter was not tidally ejected. Instead, it was ejected from the remnant during merger, but still orbiting it, when a shock wave originating from the remnant oscillations finally liberated the material. We validated this picture by tracking ejecta trajectories backward in time, in the same fashion described for the matter trajectories in the orbital plane, but using 3D data. Using this method, we also found that the average ejecta temperature was increased from $\approx 1 \mathrm{MeV}$ to $\approx 2.5 \mathrm{MeV}$ when receiving the final kick that liberated the matter. The specific entropy was increased as well, which implies shock heating. Subsequently, the ejecta cooled down again adiabatically. The implications for nucleosynthesis will be discussed in a future publication; here we just recall that the initial temperature might be important for the final abundances of heavy elements produced by the r-process nucleosynthesis. At the time the ejected material became classified as unbound according to the geodesic criterion, the temperature was already decreased by adiabatic expansion. It therefore seems prudent to track ejected material back in time to get the full thermal history.

\section{SUMMARY AND CONCLUSIONS}

We evolved the merger of binaries consisting of two $1.4 M_{\odot}$ NSs with different combinations of aligned and antialigned initial NS spins, as well as the irrotational case. Our models employ the Shen-Horowitz-Teige EOS, for which the merger at the given total mass results in a stable neutron star. We considered moderate rotation rates of $164 \mathrm{~Hz}$. If such rates are reached in nature for merging binaries is an open question; here we studied the possible consequences if this is the case. In particular, we investigated the inspiral time, the fluid flow in the remnant, the GW signal, and the mass ejection.

The inspiral time clearly depends on the spin. If both stars are aligned, the inspiral takes around two orbits longer than for a system where both are antialigned. The irrotational and mixed alignment cases are in between, the latter merging slightly quicker. We caution that our initial data has some residual eccentricity. However, the same trend has been observed in several other studies [9-11,19] which use completely different methods of constructing initial data with spin. In particular, the mixed system studied in [11] merged faster than the irrotational one, as in our case. As pointed out by [11], the post-Newtonian spin-orbit coupling should cancel for the mixed case (with equal absolute spin), which means the difference is due to terms accounting for the spin-spin coupling and spin self-coupling. Note however there is also the possibility that tidal effects are modified by the spin, as discussed in [81].

Regarding the remnant evolution, we find the same general picture as in [26]: in a coordinate frame corotating with the main $m=2$ density perturbation, we observe a slowly evolving pattern exhibiting a strong nonlinear density perturbation, and a fluid flow consisting initially of two large vortices which slowly merge into one vortex that could be described as strongly deformed differential rotation. However, the fluid flow also features secondary vortices that are phase locked with the main density deformation and coincide with the location of hot spots. The secondary vortices in our simulations persist at least $20 \mathrm{~ms}$; note however that we did not include magnetic fields, which, depending on the strength, might reduce the lifetime.

The spin strongly influences the size, shape and distribution of the vortices. In particular, the mixed spin case resulted in a very asymmetric pattern. The structures are not completely stationary, but change gradually towards a more axisymmetric state. For the mixed case, this change took place more rapidly, causing also a small shift of the GW frequency around $8 \mathrm{~ms}$ after merger. This case is also a hint that the rearrangement is not merely a reaction to the gradual angular momentum loss due to GWs, and that the remnant might possess a complex internal dynamics, at least in the early postmerger phase relevant to $\mathrm{GW}$ astronomy. We also analyzed the fluid flow of an unequal mass system employing the APR4 EOS, and unsurprisingly found an asymmetric vortex structure. Finally, we performed a long-term evolution of an equal mass binary with the APR4 EOS and a similar total mass, and found that $40 \mathrm{~ms}$ after merger all secondary vortices had decayed, with a fluid flow described by differential rotation. This stage is however not relevant to GW astronomy since the strain amplitude is already too small.

The overall rotation profile in the equatorial plane for all our models showed a maximum in the outer layers of the remnant, while the core rotated slowly and the matter 
further out gradually approached Kepler velocity. We also found that the maximum rotation rate is given by the rotation rate of the density deformation, which in turn determines the main GW frequency. This behavior was already found for many different models in [9,25-27] and seems to hold regardless of mass, mass ratio, EOS, and spin. It becomes increasingly clear that the cores of merger remnants typically do not rotate fast enough to have a significant impact on the radial structure, and that the stability and lifetime are determined by the evolution of the outer layers and possibly the disk. In this work, we measured the radial mass distribution based on a new measure introduced in [26] and find indeed that the core can be approximated very well by the core of a TOV solution.

The main effect of the spin on the GW signal emitted by our models was the different length of the inspiral phase. The frequency changes in the postmerger phase were smaller than the width of the main peak in the Fourier spectrum. For the mixed model, we noticed the appearance of a side peak that was apparently caused by the aforementioned rearrangement of the fluid flow.

Unrelated to our study of the spin, we also investigated a generic feature of BNS GW waveforms, namely the presence of sudden phase jumps during and/or after merger. We explain those in terms of overmodulation, i.e. we regard the GW as an amplitude-modulated signal where the modulation amplitude can have zero crossings. In the context of gravitational waves, the amplitude modulation is given by the remnant's quadrupole moment in a rotating frame, and the carrier frequency to the rotation rate of said frame, which is chosen such that the respective quadrupole moment is varying slowly. In all cases, we observed a phase jump during merger. This corresponds to an exchange of the principal axes of the mass distribution in the corotating frame when the stars collide, which is very plausible. We have demonstrated that cancellation effects due to the phase jumps can have a strong impact on the GW power spectra, at least with regard to secondary peaks, and should therefore be considered for any GW analysis in frequency space.
Gradual near-zero crossings of the quadrupole moment in the rotating frame might also explain some of the minima in the strain often found in numerical studies. For one such case we could rule out other explanations for the rebrightening of the GWs, such as unstable mode growth, since the amplitude minima was accompanied by a clear phase jump.

Finally, we studied the mass ejection and found a strong influence of the spin. The configuration with both spins aligned ejected the largest amount of matter, around $10^{-3} M_{\odot}$. Note that the influence of spin depends also on the ejection mechanism. In our case, we found a single wave of matter consisting of material ejected from the disk by a shock wave originating from the remnant. The matter was heated by the shock to around $2 \mathrm{MeV}$ and subsequently cooled by adiabatic expansion. In [9], we studied heavier models with the same EOS and found that the irrotational model ejected more matter than the aligned one. In this case, matter was ejected in several waves caused by the strong radial pulsations of the remnant, which in turn were stronger in the irrotational case. For the unequal mass systems studied in [11], the matter was ejected tidally and the amount depended on the spin of the lighter star. Comparing those studies, we conclude that the initial spin has a strong influence, but whether a given spin leads to more or less ejecta is hard to predict due to the different ejection mechanisms.

\section{ACKNOWLEDGMENTS}

We acknowledge support from MIUR FIR Grant No. RBFR13QJYF. Numerical calculations have been made possible through a CINECA ISCRA class B Grant providing access to the FERMI cluster, and a CINECAINFN agreement, providing access to resources on GALILEO and MARCONI at CINECA. We acknowledge PRACE for awarding us access to resource SUPERMUC based in Germany at LRZ (Grant No. GRSimStar). We would also like to thank the referee for many valuable suggestions.
[1] B. P. Abbott et al. (LIGO Scientific Collaboration and Virgo Collaboration), Phys. Rev. Lett. 116, 061102 (2016).

[2] B. P. Abbott et al. (LIGO Scientific Collaboration and Virgo Collaboration), Phys. Rev. Lett. 116, 241103 (2016).

[3] J. Abadie, B. P. Abbott, R. Abbott, M. Abernathy, T. Accadia, F. Acernese, C. Adams, R. Adhikari, P. Ajith, B. Allen et al., Classical Quantum Gravity 27, 173001 (2010).

[4] B. P. Abbott et al. (LIGO Scientific Collaboration and Virgo Collaboration), Astrophys. J. Lett. 832, L21 (2016).

[5] T. Hinderer, A. Taracchini, F. Foucart, A. Buonanno, J. Steinhoff, M. Duez, L. E. Kidder, H. P. Pfeiffer, M. A.
Scheel, B. Szilagyi, K. Hotokezaka, K. Kyutoku, M. Shibata, and C. W. Carpenter, Phys. Rev. Lett. 116, 181101 (2016).

[6] S. Bernuzzi, A. Nagar, T. Dietrich, and T. Damour, Phys. Rev. Lett. 114, 161103 (2015).

[7] K. Hotokezaka, K. Kyutoku, H. Okawa, and M. Shibata, Phys. Rev. D 91, 064060 (2015).

[8] W. Kastaun, F. Galeazzi, D. Alic, L. Rezzolla, and J. A. Font, Phys. Rev. D 88, 021501 (2013).

[9] W. Kastaun and F. Galeazzi, Phys. Rev. D 91, 064027 (2015). 
[10] P. Tsatsin and P. Marronetti, Phys. Rev. D 88, 064060 (2013).

[11] T. Dietrich, S. Bernuzzi, M. Ujevic, and W. Tichy, Phys. Rev. D 95, 044045 (2017).

[12] M. Burgay, N. D’Amico, A. Possenti, R. N. Manchester, A. G. Lyne, B. C. Joshi, M. A. McLaughlin, M. Kramer, J. M. Sarkissian, F. Camilo, V. Kalogera, C. Kim, and D. R. Lorimer, Nature (London) 426, 531 (2003).

[13] W. Tichy, Phys. Rev. D 84, 024041 (2011).

[14] T. Dietrich, N. Moldenhauer, N. K. Johnson-McDaniel, S. Bernuzzi, C. M. Markakis, B. Brügmann, and W. Tichy, Phys. Rev. D 92, 124007 (2015).

[15] W. E. East, V. Paschalidis, F. Pretorius, and S. L. Shapiro, Phys. Rev. D 93, 024011 (2016).

[16] V. Paschalidis, W. E. East, F. Pretorius, and S. L. Shapiro, Phys. Rev. D 92, 121502 (2015).

[17] M. Clark and P. Laguna, Phys. Rev. D 94, 064058 (2016).

[18] N. Tacik, F. Foucart, H. P. Pfeiffer, R. Haas, S. Ossokine, J. Kaplan, C. Muhlberger, M. D. Duez, L. E. Kidder, M. A. Scheel, and B. Szilágyi, Phys. Rev. D 92, 124012 (2015); 94, 049903(E) (2016).

[19] S. Bernuzzi, T. Dietrich, W. Tichy, and B. Brügmann, Phys. Rev. D 89, 104021 (2014).

[20] T. Dietrich, S. Bernuzzi, and W. Tichy, arXiv:1706.02969.

[21] W. Tichy, Phys. Rev. D 86, 064024 (2012).

[22] K. Hotokezaka, K. Kyutoku, H. Okawa, M. Shibata, and K. Kiuchi, Phys. Rev. D 83, 124008 (2011).

[23] A. Bauswein, T. W. Baumgarte, and H.-T. Janka, Phys. Rev. Lett. 111, 131101 (2013).

[24] T. W. Baumgarte, S. L. Shapiro, and M. Shibata, Astrophys. J. Lett. 528, L29 (2000).

[25] A. Endrizzi, R. Ciolfi, B. Giacomazzo, W. Kastaun, and T. Kawamura, Classical Quantum Gravity 33, 164001 (2016).

[26] W. Kastaun, R. Ciolfi, and B. Giacomazzo, Phys. Rev. D 94, 044060 (2016).

[27] M. Hanauske, K. Takami, L. Bovard, L. Rezzolla, J. A. Font, F. Galeazzi, and H. Stöcker, Phys. Rev. D 96, 043004 (2017).

[28] A. Bauswein and H.-T. Janka, Phys. Rev. Lett. 108, 011101 (2012).

[29] A. Bauswein, H.-T. Janka, K. Hebeler, and A. Schwenk, Phys. Rev. D 86, 063001 (2012).

[30] K. Hotokezaka, K. Kiuchi, K. Kyutoku, T. Muranushi, Y.-I. Sekiguchi, M. Shibata, and K. Taniguchi, Phys. Rev. D 88, 044026 (2013).

[31] A. Bauswein, N. Stergioulas, and H.-T. Janka, Phys. Rev. D 90, 023002 (2014).

[32] K. Takami, L. Rezzolla, and L. Baiotti, Phys. Rev. D 91, 064001 (2015).

[33] S. Bernuzzi, T. Dietrich, and A. Nagar, Phys. Rev. Lett. 115, 091101 (2015).

[34] A. Feo, R. D. Pietri, F. Maione, and F. Löffler, Classical Quantum Gravity 34, 034001 (2017).

[35] G. Shen, C. J. Horowitz, and S. Teige, Phys. Rev. C 82, 015806 (2010).

[36] G. Shen, C. J. Horowitz, and S. Teige, Phys. Rev. C 83, 035802 (2011).

[37] J. S. Read, B. D. Lackey, B. J. Owen, and J. L. Friedman, Phys. Rev. D 79, 124032 (2009).

[38] A. Akmal, V. R. Pandharipande, and D. G. Ravenhall, Phys. Rev. C 58, 1804 (1998).
[39] R. Ciolfi, W. Kastaun, B. Giacomazzo, A. Endrizzi, D. M. Siegel, and R. Perna, Phys. Rev. D 95, 063016 (2017).

[40] E. Gourgoulhon, P. Grandclement, K. Taniguchi, J.-A. Marck, and S. Bonazzola, Phys. Rev. D 63, 064029 (2001).

[41] LORENE code homepage, http://www.lorene.obspm.fr.

[42] A. Ashtekar and B. Krishnan, Phys. Rev. D 68, 104030 (2003).

[43] A. Ashtekar, C. Beetle, and J. Lewandowski, Phys. Rev. D 64, 044016 (2001).

[44] A. Ashtekar and B. Krishnan, Living Rev. Relativ. 7, 10 (2004).

[45] F. Galeazzi, W. Kastaun, L. Rezzolla, and J. A. Font, Phys. Rev. D 88, 064009 (2013).

[46] D. Brown, P. Diener, O. Sarbach, E. Schnetter, and M. Tiglio, Phys. Rev. D 79, 044023 (2009).

[47] F. Löffler, J. Faber, E. Bentivegna, T. Bode, P. Diener, R. Haas, I. Hinder, B. C. Mundim, C. D. Ott, E. Schnetter, G. Allen, M. Campanelli, and P. Laguna, Classical Quantum Gravity 29, 115001 (2012).

[48] D. Alic, C. Bona-Casas, C. Bona, L. Rezzolla, and C. Palenzuela, Phys. Rev. D 85, 064040 (2012).

[49] D. Alic, W. Kastaun, and L. Rezzolla, Phys. Rev. D 88, 064049 (2013).

[50] C. Bona, J. Massó, E. Seidel, and J. Stela, Phys. Rev. Lett. 75, 600 (1995).

[51] M. Alcubierre, B. Brügmann, P. Diener, M. Koppitz, D. Pollney, E. Seidel, and R. Takahashi, Phys. Rev. D 67, 084023 (2003).

[52] E. Schnetter, S. H. Hawley, and I. Hawke, Classical Quantum Gravity 21, 1465 (2004).

[53] T. Nakamura, K. Oohara, and Y. Kojima, Prog. Theor. Phys. Suppl. 90, 1 (1987).

[54] M. Shibata and T. Nakamura, Phys. Rev. D 52, 5428 (1995).

[55] T. W. Baumgarte and S. L. Shapiro, Phys. Rev. D 59, 024007 (1998).

[56] N. K. Glendenning and S. A. Moszkowski, Phys. Rev. Lett. 67, 2414 (1991).

[57] H. Müller and B. D. Serot, Nucl. Phys. A606, 508 (1996).

[58] F. Maione, R. D. Pietri, A. Feo, and F. Löffler, Classical Quantum Gravity 33, 175009 (2016).

[59] C. Reisswig and D. Pollney, Classical Quantum Gravity 28, 195015 (2011).

[60] V. Moncrief, Ann. Phys. (N.Y.) 88, 323 (1974).

[61] Q. Li and L.E. Atlas, in Advanced signal processing algorithms, architectures, and implementations XIV, Proc. SPIE 5559, 172 (2004).

[62] K. Kyutoku, M. Shibata, and K. Taniguchi, Phys. Rev. D 90, 064006 (2014).

[63] K. Kiuchi, P. Cerdá-Durán, K. Kyutoku, Y. Sekiguchi, and M. Shibata, Phys. Rev. D 92, 124034 (2015).

[64] See Supplemental Material at http://link.aps.org/ supplemental/10.1103/PhysRevD.96.043019 for (i) Movies showing entropy density and fluid trajectories, (ii) Gravitational waveforms from our simulations, and (iii) Movie showing mass density, unbound matter, and mesh refinement boundaries.

[65] N. Stergioulas, A. Bauswein, K. Zagkouris, and H.-T. Janka, Mon. Not. R. Astron. Soc. 418, 427 (2011). 
[66] F. Foucart, R. Haas, M. D. Duez, E. O’Connor, C. D. Ott, L. Roberts, L. E. Kidder, J. Lippuner, H. P. Pfeiffer, and M. A. Scheel, Phys. Rev. D 93, 044019 (2016).

[67] M. Shibata and K. Kiuchi, Phys. Rev. D 95, 123003 (2017).

[68] D. J. A. McKechan, C. Robinson, and B. S. Sathyaprakash, Classical Quantum Gravity 27, 084020 (2010).

[69] D. Shoemaker, Advanced LIGO anticipated sensitivity curves, https://dcc.ligo.org/LIGO-T0900288/public.

[70] B. P. Abbott, R. Abbott, T. D. Abbott, M. R. Abernathy, F. Acernese, K. Ackley, C. Adams, T. Adams, P. Addesso, R. X. Adhikari et al., Living Rev. Relativ. 19, 1 (2016).

[71] Advanced Virgo anticipated sensitivity curves, https://dcc .ligo.org/public/0094/P1200087/019/fig1_adv_sensitivity .txt.

[72] S. Hild, S. Chelkowski, and A. Freise, arXiv:0810.0604.

[73] Einstein telescope anticipated sensitivity curves, http://www .et-gw.eu/index.php/etsensitivities.
[74] S. Vinciguerra, M. Drago, G. A. Prodi, S. Klimenko, C. Lazzaro, V. Necula, F. Salemi, V. Tiwari, M. C. Tringali, and G. Vedovato, Classical Quantum Gravity 34, 094003 (2017).

[75] J. Clark, A. Bauswein, L. Cadonati, H.-T. Janka, C. Pankow, and N. Stergioulas, Phys. Rev. D 90, 062004 (2014).

[76] J. A. Clark, A. Bauswein, N. Stergioulas, and D. Shoemaker, Classical Quantum Gravity 33, 085003 (2016).

[77] K. Takami, L. Rezzolla, and L. Baiotti, Phys. Rev. Lett. 113, 091104 (2014).

[78] T. Dietrich, M. Ujevic, W. Tichy, S. Bernuzzi, and B. Brügmann, Phys. Rev. D 95, 024029 (2017).

[79] M. Shibata, S. Karino, and Y. Eriguchi, Mon. Not. R. Astron. Soc. 343, 619 (2003).

[80] F. Löffler, R. De Pietri, A. Feo, F. Maione, and L. Franci, Phys. Rev. D 91, 064057 (2015).

[81] P. Pani, L. Gualtieri, and V. Ferrari, Phys. Rev. D 92, 124003 (2015). 